OPEN ACCESS

Edited by:

James Cheng-Chung Wei, Chung Shan Medical University,

Taiwan

Reviewed by:

Taisei Mushiroda,

RIKEN Center for Integrative Medical

Sciences (IMS), Japan

Zhiguo Xie,

Central South University, China Xiaobing Wang,

First Affiliated Hospital of Wenzhou

Medical University, China

*Correspondence:

Chonlaphat Sukasem

chonlaphat.suk@mahidol.ac.th

Specialty section:

This article was submitted to

Pharmacogenetics and

Pharmacogenomics,

a section of the journal

Frontiers in Pharmacology

Received: 23 June 2019

Accepted: 27 January 2020

Published: 27 February 2020

Citation:

Satapornpong $P$, Jinda $P$, Jantararoungtong $T$, Koomdee $N$, Chaichan C, Pratoomwun J, Na Nakorn C, Aekplakorn W,

Wilantho A, Ngamphiw C, Tongsima S and Sukasem C (2020) Genetic Diversity of HLA Class I

and Class II Alleles in Thai

Populations: Contribution to Genotype-Guided Therapeutics.

Front. Pharmacol. 11:78. doi: 10.3389/fphar.2020.00078

\section{Genetic Diversity of HLA Class I and Class II Alleles in Thai Populations: Contribution to Genotype-Guided Therapeutics}

\author{
Patompong Satapornpong ${ }^{1,2,3}$, Pimonpan Jinda ${ }^{1,2}$, Thawinee Jantararoungtong ${ }^{1,2}$, \\ Napatrupron Koomdee ${ }^{1,2}$, Chonlawat Chaichan ${ }^{1,2}$, Jirawat Pratoomwun ${ }^{1,2}$, \\ Chalitpon Na Nakorn ${ }^{4}$, Wichai Aekplakorn ${ }^{5}$, Alisa Wilantho ${ }^{6,7}$, Chumpol Ngamphiw ${ }^{6,7}$, \\ Sissades Tongsima ${ }^{6,7}$ and Chonlaphat Sukasem ${ }^{1,2,8^{*}}$ \\ ${ }^{1}$ Division of Pharmacogenomics and Personalized Medicine, Department of Pathology, Faculty of Medicine Ramathibodi \\ Hospital, Mahidol University, Bangkok, Thailand, 2 Laboratory for Pharmacogenomics, Somdech Phra Debaratana Medical \\ Center (SDMC), Ramathibodi Hospital, Bangkok, Thailand, ${ }^{3}$ Division of General Pharmacy Practice, Department of \\ Pharmaceutical Care, College of Pharmacy, Rangsit University, Pathum Thani, Thailand, ${ }^{4}$ Graduate Program in Translational \\ Medicine, Research Center, Faculty of Medicine Ramathibodi Hospital, Mahidol University, Bangkok, Thailand, ${ }^{5}$ Department \\ of Community Medicine, Faculty of Medicine Ramathibodi Hospital, Mahidol University, Bangkok, Thailand, ${ }^{6}$ National \\ Biobank of Thailand, National Science and Technology Development Agency, Pathum Thani, Thailand, ${ }^{7}$ National Center for \\ Genetic Engineering and Biotechnology, National Science and Technology Development Agency, Pathum Thani, Thailand, \\ ${ }^{8}$ The Thai Severe Cutaneous Adverse Drug Reaction (THAI-SCAR) Research Group, Bangkok, Thailand
}

Human leukocyte antigen (HLA) class I and II are known to have association with severe cutaneous adverse reactions (SCARs) when exposing to certain drug treatment. Due to genetic differences at population level, drug hypersensitivity reactions are varied, and thus common pharmacogenetics markers for one country might be different from another country, for instance, $H L A-A^{*} 31: 01$ is associated with carbamazepine (CBZ)-induced SCARs in European and Japanese while HLA-B*15:02 is associated with CBZ-induced Stevens-Johnson syndrome/toxic epidermal necrolysis (SJS/TEN) among Taiwanese and Southeast Asian. Such differences pose a major challenge to prevent drug hypersensitivity when pharmacogenetics cannot be ubiquitously and efficiently translated into clinic. Therefore, a population-wide study of the distribution of HLA-pharmacogenetics markers is needed. This work presents a study of Thai HLA alleles on both HLA class I and II genes from 470 unrelated Thai individuals by means of polymerase chain reaction sequence-specific oligonucleotide (PCR-SSO) in which oligonucleotide probes along the stretches of $H L A-A,-B,-C,-D R B 1,-D Q A 1$, and $-D Q B 1$ genes were genotyped. These 470 individuals were selected according to their regional locations, which were from North, Northeast, South, Central, and a capital city, Bangkok. Top ranked HLA alleles in Thai population include HLA-A*11:01 (26.06\%), - $B^{*} 46: 01$ (14.04\%), $-C^{*} 01: 02$ (17.13\%), $-D R B 1 * 12: 02$ (15.32\%), $-D Q A 1{ }^{*} 01: 01$ (24.89\%), and $-D Q B 1{ }^{*} 05: 02$ (21.28\%). The results revealed that the distribution of HLA-pharmacogenetics alleles from the South had more HLA-B75 family that a typical HLA-B*15:02 pharmacogenetics test for SJS/TEN screening would not cover. Besides the view across the nation, when compared HLA 
alleles from Thai population with HLA alleles from both European and Asian countries, the distribution landscape of HLA-associated drug hypersensitivity across many countries could be observed. Consequently, this pharmacogenetics database offers a comprehensive view of pharmacogenetics marker distribution in Thailand that could be used as a reference for other Southeast Asian countries to validate the feasibility of their future pharmacogenetics deployment.

\section{Keywords: human leukocyte antigen, HLA class I, HLA class II, Thai population, pharmacogenetic marker}

\section{INTRODUCTION}

Human leukocyte antigen (HLA) gene is located on chromosome $6 \mathrm{p} 21$, which was considered the most polymorphic of human genetic system (Shiina et al., 2009). HLA encodes cell surface molecules that present antigenic peptides to the $\mathrm{T}$-cell receptor (TCR) on T cells (Sette and Sidney, 1998). There are two main classes of HLA allele. HLA class I and II encode cell surface heterodimers that play a role in antigen presentation, tolerance, and self/nonself-recognition. HLA class I molecules gather peptides that have been synthesized within the individual nucleated cell, three main $H L A$ class I genes including $H L A-A$, $H L A-B$, and HLA-C (Howell et al., 2010). Whereas HLA class II molecules gather exogenously synthesized peptide ligands by endocytic pathway and expressed with antigen-presenting cells (APCs), six main HLA class II genes including HLA-DPA1, HLADPB1, HLA-DQA1, HLA-DQB1, HLA-DRA, and HLA-DRB1 (Ulvestad et al., 1994). The HLA system plays a critical role in regulating the immune response, tissue or organ transplantation, autoimmunity, vaccine development, susceptibility or resistance disease, and pharmacogenomics (Anania et al., 2017; Illing et al., 2017; Petersdorf, 2017). Over the past decade, there have been reported associations between various $H L A$ alleles and different adverse drug reactions, especially severe cutaneous adverse reactions (SCARs).

One prominent report on drug-induced SCARs is the association between $H L A-B^{\star}$ 15:02 allele and carbamazepine (CBZ)-induced Stevens-Johnson syndrome/toxic epidermal necrolysis (SJS/TEN) in Han Chinese, Thai, and Southeast Asians (Ferrell and McLeod, 2008; Locharernkul et al., 2008; Hung et al., 2010; Chang et al., 2011; Nguyen et al., 2015). Conversely, Japanese and European populations were shown to have the association of $H L A-A A^{\star 31: 01}$ and $\mathrm{CBZ}$-induced hypersensitivity reactions (McCormack et al., 2011; Ozeki et al., 2011). While HLA-B*58:01 could be used as a pharmacogenetic risk prediction marker for allopurinolinduced SJS/TEN in many populations (Somkrua et al., 2011). In addition, the association between HLA class II and adverse drug reactions were reported, such as amoxicillin-clavulanate that induces liver injury was found to be associated with $H L A$ $D R B 1^{\star} 15: 01, H L A-D R B 5^{\star} 01: 01$, and $H L A-D Q B 1^{\star} 06: 02$ haplotype in European (Hautekeete et al., 1999; Lucena et al., 2011). Thus, the distributions of HLA alleles and pharmacogenetic markers that could vary among different populations might affect incidences of adverse drug reactions or drug dosage responses (Donnell PH and Dolan, 2009).
Although there is a clear need to investigate at a population level HLA alleles, a study on a distribution of Thai HLA alleles was limited. Puangpetch et al. (2015) previously reported only $H L A-B$ polymorphisms from 986 Thai individuals. The top five of such $H L A-B$ alleles consisted of $H L A-B^{\star} 46: 01$ (11.51\%), HLA$B^{\star} 58: 01$ (8.62\%), HLA-B ${ }^{\star} 40: 01$ (8.22\%), HLA- $B^{\star} 15: 02$ (8.16\%), and $H L A-B^{\star} 13: 01$ (6.95\%). However, from this work, there were no reports of $H L A$ class I and II allele in Thai population. There are other HLA alleles that play important roles in predicting various adverse drug reactions. Hence, the aim of this study was to comprehensively investigate both $H L A$ class I ( $H L A-A,-B$, and $-C)$ and II (HLA-DRB1, $-D Q A 1$, and $-D Q B 1)$ distribution of alleles in Thailand and the potential association with adverse drug reactions of these alleles.

\section{MATERIALS AND METHODS}

\section{Subjects}

We recruited 470 unrelated healthy Thai individuals from the 4th National Health Examination Survey in Thailand during August 2008 and March 2009, and the information was obtained from National Health Examination Survey Office, Health System Research Institute, Ministry of Public Health, Thailand. The 470 Thai individuals were randomly chosen according to their selfreported origins which can be characterized into five regional groups: $(\mathrm{n}=70)$ Bangkok, $(\mathrm{n}=100)$ Central, $(\mathrm{n}=100)$ Northeastern, $(n=100)$ Northern, and $(n=100)$ Southern. Since we want this study to represent majority of Thai people, subjects for each group must have lived in the aforementioned regions for more than three generations. Furthermore, these healthy individuals must have no history of cutaneous adverse drug reactions (CADRs). Thailand is a country located at the center of Southeast Asia, sharing boundaries with Myanmar (west), Laos (north east), Cambodia (east), and Malaysia (south). This study was approved by the Ethical Review Committee on Research Involving Human Subjects, Faculty of Medicine, Ramathibodi Hospital, Mahidol University. Written informed consent was obtained from all participants.

\section{HLA Class I and II Genotyping}

Recruited genomic DNA samples were isolated from EDTA blood using the MagNAprue Compact Nucleic Acid Isolation kits (Roche Applied Science, Mannheim, Germany). The quality of genomic DNA was measured by NanoDrop ${ }^{\circledR}$ ND-1000 
(Thermo Scientific, Wilmington, USA). HLA class I alleles, comprising $H L A-A, H L A-B$, and $H L A-C$, and $H L A$ class II alleles, comprising $H L A-D R B 1$, HLA-DQA1, and $H L A-D Q B 1$, were genotyped using sequence-specific oligonucleotides (PCRSSOs). Briefly, the DNA samples obtained from patients of the five regions in Thailand were amplified by polymerase chain reaction (PCR). The PCR products were hybridized against a panel of SSO probes on coated polystyrene microspheres that had sequences complementary to the stretches of polymorphism within the target HLA class I and II alleles using the Lifecodes HLA SSO typing kits (Immucor, West Avenue, Stamford, USA). The amplicon-probe complex was then visualized using a colorimetric reaction and fluorescence detection technology by the Luminex ${ }^{\circledR}$ IS 100 system (Luminex Corporation, Austin, Texas, USA). Interpretations of HLA class I and II alleles from the probe signals were performed using MATCH IT DNA software version 1.2.2 (One Lambda, Canoga Park, CA, USA).

\section{Statistical Analysis}

The allele frequency and statistical analyses were performed using the Arlequin program version 3.1 for Hardy-Weinberg equilibrium testing. We used SPSS Compare allele frequencies between each region in Thailand using the SPSS software for Windows version 16.0 (SPSS Inc., Chicago, IL). A given pair of each region in Thai population was determined significant difference if the $p$-value was less than 0.05 .

\section{Population Structure Analysis}

There were only six HLA haplotypes from class I and II to be used in this population structure analysis which were not enough to investigate substructure from these 470 individuals. We employed HLA probe polymorphism signals obtained directly from each "stretch" of the six HLA haplotypes. The total number of probes used in this experiment was 403 polymorphism probes distributed across six $H L A$ haplotypes. The numbers of probes for six $H L A$ haplotypes are as follows: $H L A-A$ (72 probes), HLA$B$ (92 probes), and $H L A-C$ (77 probes) for HLA class I and HLADRB1 (91 probes), HLA-DQA1 (23 probes), and HLA-DQB1 (48 probes) for HLA class II.

We concatenated the raw data containing probe signals for each HLA-haplotype into one tab-delimited file (Supplemental text: mergeallHLA.txt) containing a 470403 HLA-probe matrix that was used in both principal component analysis (PCA) (Chaichompoo et al., 2018) and STRUCTURE analysis (Pritchard et al., 2000; Kopelman et al., 2015).

\section{Principal Component Analysis}

Before performing PCA, the HLA-probe data entries were normalized based on $\mathrm{Z}$-score calculation. In particular, a probe signal $X$ is converted to $X^{\prime}=(X-\bar{X}) / \mathrm{SD}$ where SD represents a standard deviation value of each probe column. The normalization step was done to minimize HLA-typing batch effects. We used cal.pclinear function with default options from KRIS R package version 1.1.1 (Chaichompoo et al., 2018) to perform PCA. The PCA visualization was done using command plot3views from KRIS R package to display three main PCA perspectives, namely, PC1 vs. $\mathrm{PC} 2, \mathrm{PC} 2$ vs. PC3, and PC1 vs. PC3.

\section{STRUCTURE Analysis}

The normalization of the raw probe data was done similar to PCA with an extra step to round all $X^{\prime}$ values into integer. The conversion was done so that STRUCTURE could treat these normalized signals as a type of variation patterns similar to that of microsatellites. For STRUCTURE analysis, we used 30,000 burnin length with 80,000 MCMC iterations. The analyses were done from $K=1$ to $K=10$ ( $K$ represents a number of Bayesianinferred clusters in a given population) each of which had 30 repeats with the same parameter setting. The total 100 STRUCTURE analysis results were used as the input to CLUMPAK (Kopelman et al., 2015), which helped determine the optimal K based on an ad hoc quantity Delta (K) approach (Evanno et al., 2005). The STRUCTURE visualization was done using pophelper function from pophelper $\mathrm{R}$ package version 2.30 (Francis, 2017).

\section{RESULTS}

\section{HLA Class I and II Allele Frequencies in Thai Population}

The allele frequency distribution of $H L A$ class I and II are shown in Tables 1-6. We found that the alleles $H L A-A^{\star} 11: 01,-A^{\star} 24: 02$, $-A^{\star} 02: 03,-A^{\star} 33: 03,-A^{\star} 02: 07$, and $-A^{\star} 02: 01$ were more common than the others. $H L A-A^{\star} 11: 01$ was the most common allele found across five designated regions. For $H L A-B, H L A-B^{\star} 46: 01$ was the predominant allele commonly found in Northern, Northeastern, Central, and Bangkok regions. $H L A-B$ allele profile from the Southern group, however, showed that $H L A-B^{\star}$ 15:02 was found more commonly (Table 2). Additionally, the allele distribution of $H L A-B$ and their frequencies were $H L A-B^{\star} 46: 01$ (14.04\%), $-B^{\star} 15: 02$ (7.66\%), $-B^{\star} 40: 01$ (6.60\%), $-B^{\star 58: 01}(6.38 \%),-B^{\star} 13: 01$ (5.96\%), $-B^{\star} 44: 03$ (4.47\%), and $-B^{\star 38: 02}$ (4.26\%), respectively. For $H L A-C$, the allele distribution and the corresponding frequencies were $H L A-C^{\star} 01: 02(17.13 \%),-C^{\star} 07: 02(11.91 \%)$, $-C^{\star} 08: 01(10.32 \%),-C^{\star} 03: 04(8.09 \%),-C^{\star} 03: 02(7.77 \%)$, $-C^{\star} 07: 01$ (6.38\%), and $-C^{\star} 07: 04(7.00 \%)$, respectively (Table 3). Particularly, $H L A-C^{\star} 08: 01$ (14.50\%) allele was the highest frequency in the Southern group, but there was not significant difference when compared with the other regions ( $p$-value $=$ 0.4764) as shown in Table 7.

The frequency of $H L A$ class II alleles including $H L A-D R B 1$, $H L A-D Q A 1$, and HLA-DQB1 alleles were presented in Tables 4 6. The frequency distributions of $H L A-D R B 1$ alleles were $H L A$ $D R B 1^{\star} 12: 02$ (15.32\%), $-D R B 1^{\star} 15: 02(14.47 \%),-D R B 1^{\star} 09: 01$ (9.89\%), $-D R B 1^{\star} 07: 01 \quad(8.94 \%),-D R B 1^{\star} 15: 01 \quad(8.09 \%)$, $-D R B 1^{\star} 14: 01$ (5.96\%), $-D R B 1^{\star} 16: 02$ (5.96\%), and $-D R B 1^{\star 03: 01}$ (5.00\%). Particularly, HLA-DRB1*15:02 was the highest allele frequency presented in the Southern, Northern, and Northeastern groups, while Central and Bangkok regions share the top allele frequency of $H L A-D R B 1^{*} 12: 02$. Moreover, frequencies of $H L A-D Q A 1$ alleles were $H L A-D Q A 1^{\star} 01: 01$ (24.89\%), $-D Q A 1^{\star} 01: 02(22.23 \%),-D Q A 1^{\star} 03: 02(13.30 \%)$, -DQA $1^{*} 06: 01(12.02 \%),-D Q A 1^{*} 02: 01 \quad(8.72 \%)$, and $-D Q A 1^{\star} 05: 01$ (5.43\%) (Table 5). HLA-DQA1*01:01 allele was 
TABLE 1 | The frequency of HLA class I alleles in Thai population and five regions $(n=470)$.

\begin{tabular}{|c|c|c|c|c|c|c|c|c|c|c|c|c|c|c|c|c|c|c|}
\hline \multirow[t]{2}{*}{ HLA-A } & \multicolumn{3}{|c|}{ Thailand $(n=470)$} & \multicolumn{3}{|c|}{ Southern $(n=100)$} & \multicolumn{3}{|c|}{ Northern $(n=100)$} & \multicolumn{3}{|c|}{ Northeastern $(n=100)$} & \multicolumn{3}{|c|}{ Central $(n=100)$} & \multicolumn{3}{|c|}{ Bangkok $(n=70)$} \\
\hline & AF & CF & $\underset{\text { (p-value) }}{\text { HW }}$ & AF & CF & $\underset{\text { (p-value) }}{\text { HW }}$ & AF & CF & $\underset{\text { (p-value) }}{\mathrm{HW}}$ & AF & CF & $\begin{array}{c}\text { HW } \\
\text { (p-value) }\end{array}$ & AF & CF & $\begin{array}{c}\text { HW } \\
\text { (p-value) }\end{array}$ & AF & CF & $\begin{array}{c}\text { HW } \\
\text { (p-value) }\end{array}$ \\
\hline 01:01 & 0.0223 & 0.04468 & 1.0000 & 0.0450 & 0.0900 & 1.0000 & 0.0050 & 0.0100 & 1.0000 & 0.0050 & 0.0100 & 1.0000 & 0.0250 & 0.0500 & 1.0000 & 0.0357 & 0.0714 & 1.0000 \\
\hline 01:03 & 0.0011 & 0.00213 & 1.0000 & & & & & & & & & & & & & 0.0071 & 0.0143 & 1.0000 \\
\hline 02:01 & 0.0553 & 0.10851 & 1.0000 & 0.0450 & 0.0800 & 1.0000 & 0.0400 & 0.0800 & 1.0000 & 0.0350 & 0.0700 & 1.0000 & 0.0700 & 0.1400 & 1.0000 & 0.1000 & 0.2000 & 1.0000 \\
\hline 02:02 & 0.0011 & 0.00213 & 1.0000 & & & & 0.0050 & 0.0100 & 1.0000 & & & & & & & & & \\
\hline 02:03 & 0.1117 & 0.21064 & 1.0000 & 0.0400 & 0.0800 & 1.0000 & 0.1450 & 0.2800 & 1.0000 & 0.1400 & 0.2400 & 0.6827 & 0.1350 & 0.2600 & 1.0000 & 0.0929 & 0.1857 & 1.0000 \\
\hline 02:06 & 0.0223 & 0.04468 & 1.0000 & 0.0200 & 0.0400 & 1.0000 & 0.0200 & 0.0400 & 1.0000 & 0.0300 & 0.0600 & 1.0000 & 0.0250 & 0.0500 & 1.0000 & 0.0143 & 0.0286 & 1.0000 \\
\hline 02:07 & 0.0840 & 0.14468 & 0.0556 & 0.0500 & 0.0900 & 1.0000 & 0.1000 & 0.1700 & 1.0000 & 0.1000 & 0.1800 & 1.0000 & 0.0750 & 0.1300 & 1.0000 & 0.1000 & 0.1571 & 0.6193 \\
\hline $02: 11$ & 0.0043 & 0.00851 & 1.0000 & 0.0100 & 0.0200 & 1.0000 & 0.0050 & 0.0100 & 1.0000 & 0.0050 & 0.0100 & 1.0000 & & & & & & \\
\hline 02:17 & 0.0043 & 0.00851 & 1.0000 & 0.0050 & 0.0100 & 1.0000 & & & & & & & 0.0100 & 0.0200 & 1.0000 & 0.0071 & 0.0143 & 1.0000 \\
\hline $02: 20$ & 0.0011 & 0.00213 & 1.0000 & & & & & & & 0.0050 & 0.0100 & 1.0000 & & & & & & \\
\hline 02:33 & 0.0032 & 0.00426 & 1.0000 & & & & & & & 0.0050 & 0.0100 & 1.0000 & 0.0100 & 0.0100 & 1.0000 & & & \\
\hline 02:58 & 0.0117 & 0.02340 & 1.0000 & 0.0050 & 0.0100 & 1.0000 & 0.0150 & 0.0300 & 1.0000 & & & & 0.0250 & 0.0500 & 1.0000 & 0.0143 & 0.0286 & 1.0000 \\
\hline 03:01 & 0.0064 & 0.01277 & 1.0000 & 0.0150 & 0.0300 & 1.0000 & & & & 0.0050 & 0.0100 & 1.0000 & 0.0050 & 0.0100 & 1.0000 & 0.0071 & 0.0143 & 1.0000 \\
\hline 03:02 & 0.0011 & 0.00213 & 1.0000 & & & & & & & 0.0050 & 0.0100 & 1.0000 & & & & & & \\
\hline $11: 01$ & 0.2606 & 0.44894 & 0.8984 & 0.2300 & 0.4100 & 1.0000 & 0.3550 & 0.5900 & 0.8307 & 0.2500 & 0.4600 & 0.7475 & 0.2600 & 0.4200 & 0.6162 & 0.1857 & 0.3286 & 1.0000 \\
\hline 11:02 & 0.0181 & 0.03617 & 1.0000 & 0.0200 & 0.0400 & 1.0000 & 0.0300 & 0.0600 & 1.0000 & 0.0250 & 0.0500 & 1.0000 & 0.0050 & 0.0100 & 1.0000 & 0.0071 & 0.0143 & 1.0000 \\
\hline 11:03 & 0.0032 & 0.00638 & 1.0000 & 0.0050 & 0.0100 & 1.0000 & & & & & & & 0.0050 & 0.0100 & 1.0000 & 0.0071 & 0.0143 & 1.0000 \\
\hline 11:04 & 0.0053 & 0.01064 & 1.0000 & 0.0150 & 0.0300 & 1.0000 & & & & 0.0050 & 0.0100 & 1.0000 & & & & 0.0071 & 0.0143 & 1.0000 \\
\hline 11:09 & 0.0011 & 0.00213 & 1.0000 & 0.0050 & 0.0100 & 1.0000 & & & & & & & & & & & & \\
\hline 23:01 & 0.0011 & 0.00213 & 1.0000 & 0.0050 & 0.0100 & 1.0000 & & & & & & & & & & & & \\
\hline 24:01 & 0.0011 & 0.00213 & 1.0000 & 0.0050 & 0.0100 & 1.0000 & & & & & & & & & & & & \\
\hline 24:02 & 0.1149 & 0.20213 & 0.1643 & 0.1450 & 0.2200 & 0.1679 & 0.1000 & 0.1900 & 1.0000 & 0.1150 & 0.2100 & 1.0000 & 0.1000 & 0.1900 & 1.0000 & 0.1143 & 0.2000 & 1.0000 \\
\hline 24:03 & 0.0053 & 0.01064 & 1.0000 & & & & & & & 0.0100 & 0.0200 & 1.0000 & 0.0050 & 0.0100 & & 0.0143 & 0.0286 & 1.0000 \\
\hline 24:07 & 0.0426 & 0.08298 & 1.0000 & 0.0700 & 0.1400 & 1.0000 & 0.0300 & 0.0600 & 1.0000 & 0.0250 & 0.0500 & 1.0000 & 0.0350 & 0.0700 & 1.0000 & 0.0571 & 0.1000 & 1.0000 \\
\hline $24: 10$ & 0.0170 & 0.03404 & 1.0000 & 0.0100 & 0.0200 & 1.0000 & 0.0100 & 0.0200 & 1.0000 & 0.0400 & 0.0800 & 1.0000 & 0.0050 & 0.0100 & 1.0000 & 0.0214 & 0.0429 & 1.0000 \\
\hline $24: 17$ & 0.0011 & 0.00213 & 1.0000 & 0.0050 & 0.0100 & 1.0000 & & & & & & & & & & & & \\
\hline $24: 25$ & 0.0011 & 0.00213 & 1.0000 & 0.0050 & 0.0100 & 1.0000 & & & & & & & & & & & & \\
\hline 26:01 & 0.0128 & 0.02553 & 1.0000 & 0.0150 & 0.0300 & 1.0000 & 0.0200 & 0.0400 & 1.0000 & 0.0050 & 0.0100 & 1.0000 & 0.0150 & 0.0300 & 1.0000 & 0.0071 & 0.0143 & 1.0000 \\
\hline 29:01 & 0.0053 & 0.01064 & 1.0000 & & & & 0.0050 & 0.0100 & 1.0000 & 0.0150 & 0.0300 & 1.0000 & 0.0050 & 0.0100 & 1.0000 & & & \\
\hline 29:10 & 0.0011 & 0.00213 & 1.0000 & & & & & & & & & & & & & 0.0071 & 0.0143 & 1.0000 \\
\hline $30: 01$ & 0.0213 & 0.04255 & 1.0000 & 0.0350 & 0.0700 & 1.0000 & 0.0100 & 0.0200 & 1.0000 & 0.0200 & 0.0400 & 1.0000 & 0.0250 & 0.0500 & 1.0000 & 0.0143 & 0.0286 & 1.0000 \\
\hline $31: 01$ & 0.0085 & 0.01489 & 1.0000 & 0.0200 & 0.0300 & 1.0000 & 0.0050 & 0.0100 & 1.0000 & & & & 0.0050 & 0.0100 & 1.0000 & 0.0143 & 0.0286 & 1.0000 \\
\hline 32:01 & 0.0021 & 0.00426 & 1.0000 & 0.0050 & 0.0100 & 1.0000 & & & & & & & 0.0050 & 0.0100 & 1.0000 & & & \\
\hline 33:01 & 0.0032 & 0.00638 & 1.0000 & & & & 0.0050 & 0.0100 & 1.0000 & & & & 0.0050 & 0.0100 & 1.0000 & 0.0071 & 0.0143 & 1.0000 \\
\hline 33:03 & 0.1117 & 0.21064 & 1.0000 & 0.1050 & 0.1900 & 1.0000 & 0.0850 & 0.1700 & 1.0000 & 0.1000 & 0.1900 & 1.0000 & 0.1450 & 0.2600 & 1.0000 & 0.1286 & 0.2571 & 1.0000 \\
\hline 33:12 & 0.0021 & 0.00426 & 1.0000 & 0.0100 & 0.0200 & 1.0000 & & & & & & & & & & & & \\
\hline 34:01 & 0.0096 & 0.01915 & 1.0000 & 0.0200 & 0.0400 & 1.0000 & 0.0050 & 0.0100 & 1.0000 & 0.0150 & 0.0300 & 1.0000 & & & & 0.0071 & 0.0143 & 1.0000 \\
\hline $68: 01$ & 0.0096 & 0.01915 & 1.0000 & 0.0200 & 0.0400 & 1.0000 & & & & 0.0150 & 0.0300 & 1.0000 & & & & 0.0143 & 0.0286 & 1.0000 \\
\hline 68:02 & 0.0021 & 0.00426 & 1.0000 & 0.0100 & 0.0200 & 1.0000 & & & & & & & & & & & & \\
\hline 74:01 & 0.0064 & 0.01277 & 1.0000 & 0.0050 & 0.0100 & 1.0000 & & & & 0.0200 & 0.0400 & 1.0000 & & & & 0.0071 & 0.0143 & 1.0000 \\
\hline 74:05 & 0.0021 & 0.00426 & 1.0000 & & & & 0.0050 & 0.0100 & 1.0000 & 0.0050 & 0.0100 & 1.0000 & & & & & & \\
\hline
\end{tabular}

HLA-A, human leukocyte antigen-A; AF, allele frequency; CF, carrier frequency; HW, Hardy-Weinberg equilibrium; Significantly different p-value $<0.05$. 
TABLE 2 | The frequency of HLA class I alleles in Thai population and five regions $(n=470)$.

\begin{tabular}{|c|c|c|c|c|c|c|c|c|c|c|c|c|c|c|c|c|c|c|}
\hline \multirow[t]{2}{*}{ HLA-B } & \multicolumn{3}{|c|}{ Thailand $(n=470)$} & \multicolumn{3}{|c|}{ Southern $(n=100)$} & \multicolumn{3}{|c|}{ Northern $(n=100)$} & \multicolumn{3}{|c|}{ Northeastern $(n=100)$} & \multicolumn{3}{|c|}{ Central $(n=100)$} & \multicolumn{3}{|c|}{ Bangkok $(n=70)$} \\
\hline & AF & CF & $\begin{array}{c}\text { HW } \\
\text { (p-value) }\end{array}$ & AF & CF & $\begin{array}{c}\text { HW } \\
\text { (p-value) }\end{array}$ & AF & CF & $\begin{array}{c}\text { HW } \\
\text { (p-value) }\end{array}$ & AF & CF & $\underset{\text { (p-value) }}{\mathrm{HW}}$ & AF & CF & $\begin{array}{c}\text { HW } \\
\text { (p-value) }\end{array}$ & AF & CF & $\begin{array}{c}\text { HW } \\
\text { (p-value) }\end{array}$ \\
\hline 03:01 & 0.0011 & 0.0021 & 1.0000 & 0.0050 & 0.0100 & 1.0000 & & & & & & & & & & & & \\
\hline 03:02 & 0.0011 & 0.0021 & 1.0000 & 0.0050 & 0.0100 & 1.0000 & & & & & & & & & & & & \\
\hline 07:02 & 0.0032 & 0.0064 & 1.0000 & 0.0100 & 0.0200 & 1.0000 & & & & & & & 0.0050 & 0.0100 & 1.0000 & & & \\
\hline 07:05 & 0.0255 & 0.0511 & 1.0000 & 0.0050 & 0.0100 & 1.0000 & 0.0250 & 0.0500 & 1.0000 & 0.0400 & 0.0800 & 1.0000 & 0.0300 & 0.0600 & 1.0000 & 0.0286 & 0.0571 & 1.0000 \\
\hline 08:01 & 0.0032 & 0.0064 & 1.0000 & 0.0050 & 0.0100 & 1.0000 & & & & & & & 0.0050 & 0.0100 & 1.0000 & 0.0071 & 0.0143 & 1.0000 \\
\hline 13:01 & 0.0596 & 0.1149 & 1.0000 & 0.0350 & 0.0700 & 1.0000 & 0.0800 & 0.1400 & 0.4980 & 0.0600 & 0.1200 & 1.0000 & 0.0650 & 0.1300 & 1.0000 & 0.0571 & 0.1143 & 1.0000 \\
\hline 13:02 & 0.0213 & 0.0426 & 1.0000 & 0.0350 & 0.0700 & 1.0000 & 0.0150 & 0.0300 & 1.0000 & 0.0150 & 0.0300 & 1.0000 & 0.0150 & 0.0300 & 1.0000 & 0.0286 & 0.0571 & 1.0000 \\
\hline 13:10 & 0.0011 & 0.0021 & 1.0000 & & & & 0.0050 & 0.0100 & 1.0000 & & & & & & & & & \\
\hline 14:02 & 0.0011 & 0.0021 & 1.0000 & & & & & & & & & & & & & 0.0071 & 0.0143 & 1.0000 \\
\hline 15:01 & 0.0053 & 0.0106 & 1.0000 & 0.0050 & 0.0100 & 1.0000 & 0.0100 & 0.0200 & 1.0000 & 0.0100 & 0.0200 & 1.0000 & & & & & & \\
\hline 15:02 & 0.0766 & 0.1511 & 1.0000 & 0.0900 & 0.1800 & 1.0000 & 0.0650 & 0.1300 & 1.0000 & 0.0650 & 0.1200 & 1.0000 & 0.0750 & 0.1500 & 1.0000 & 0.0929 & 0.1857 & 1.0000 \\
\hline $15: 04$ & 0.0011 & 0.0021 & 1.0000 & 0.0050 & 0.0100 & 1.0000 & & & & & & & & & & & & \\
\hline 15:07 & 0.0021 & 0.0043 & 1.0000 & & & & 0.0100 & 0.0200 & 1.0000 & & & & & & & & & \\
\hline 15:10 & 0.0021 & 0.0043 & 1.0000 & 0.0050 & 0.0100 & 1.0000 & & & & 0.0050 & 0.0100 & 1.0000 & & & & & & \\
\hline $15: 11$ & 0.0021 & 0.0043 & 1.0000 & 0.0050 & 0.0100 & 1.0000 & 0.0050 & 0.0100 & 1.0000 & & & & & & & & & \\
\hline 15:12 & 0.0021 & 0.0043 & 1.0000 & & & & 0.0050 & 0.0100 & 1.0000 & 0.0050 & 0.0100 & 1.0000 & & & & & & \\
\hline $15: 13$ & 0.0096 & 0.0191 & 1.0000 & 0.0250 & 0.0500 & 1.0000 & & & & 0.0050 & 0.0100 & 1.0000 & 0.0100 & 0.0200 & 1.0000 & 0.0071 & 0.0143 & 1.0000 \\
\hline 15:17 & 0.0043 & 0.0085 & 1.0000 & 0.0100 & 0.0200 & 1.0000 & & & & & & & 0.0050 & 0.0100 & 1.0000 & 0.0071 & 0.0143 & 1.0000 \\
\hline $15: 18$ & 0.0032 & 0.0064 & 1.0000 & 0.0050 & 0.0100 & 1.0000 & & & & & & & & & & 0.0143 & 0.0286 & 1.0000 \\
\hline $15: 20$ & 0.0011 & 0.0021 & 1.0000 & & & & 0.0050 & 0.0100 & 1.0000 & & & & & & & & & \\
\hline $15: 21$ & 0.0021 & 0.0043 & 1.0000 & 0.0050 & 0.0100 & 1.0000 & & & & 0.0050 & 0.0100 & 1.0000 & & & & & & \\
\hline $15: 25$ & 0.0223 & 0.0426 & 1.0000 & 0.0200 & 0.0400 & 1.0000 & 0.0250 & 0.0500 & 1.0000 & 0.0300 & 0.0600 & 1.0000 & 0.0100 & 0.0200 & 1.0000 & 0.0286 & 0.0429 & 1.0000 \\
\hline 15:31 & 0.0021 & 0.0043 & 1.0000 & 0.0100 & 0.0200 & 1.0000 & & & & & & & & & & & & \\
\hline 15:32 & 0.0043 & 0.0085 & 1.0000 & 0.0100 & 0.0200 & 1.0000 & & & & 0.0100 & 0.0200 & 1.0000 & & & & & & \\
\hline 15:35 & 0.0032 & 0.0064 & 1.0000 & 0.0050 & 0.0100 & 1.0000 & 0.0050 & 0.0100 & 1.0000 & 0.0050 & 0.0100 & 1.0000 & & & & & & \\
\hline 18:01 & 0.0383 & 0.0766 & 1.0000 & 0.0500 & 0.1000 & 1.0000 & 0.0150 & 0.0300 & 1.0000 & 0.0300 & 0.0600 & 1.0000 & 0.0600 & 0.1200 & 1.0000 & 0.0357 & 0.0714 & 1.0000 \\
\hline 18:02 & 0.0160 & 0.0319 & 1.0000 & 0.0200 & 0.0400 & 1.0000 & 0.0100 & 0.0200 & 1.0000 & 0.0400 & 0.0800 & 1.0000 & & & & 0.0071 & 0.0143 & 1.0000 \\
\hline 18:12 & 0.0011 & 0.0021 & 1.0000 & & & & 0.0050 & 0.0100 & 1.0000 & & & & & & & & & \\
\hline $18: 14$ & 0.0011 & 0.0021 & 1.0000 & 0.0050 & 0.0100 & 1.0000 & & & & & & & & & & & & \\
\hline 27:03 & 0.0011 & 0.0021 & 1.0000 & & & & 0.0050 & 0.0100 & 1.0000 & & & & & & & & & \\
\hline 27:04 & 0.0213 & 0.0404 & 1.0000 & 0.0200 & 0.0400 & 1.0000 & 0.0400 & 0.0700 & 1.0000 & 0.0150 & 0.0300 & 1.0000 & 0.0100 & 0.0200 & 1.0000 & 0.0214 & 0.0429 & 1.0000 \\
\hline 27:06 & 0.0138 & 0.0255 & 1.0000 & 0.0050 & 0.0100 & 1.0000 & 0.0200 & 0.0300 & 1.0000 & 0.0350 & 0.0700 & 1.0000 & 0.0050 & 0.0100 & 1.0000 & & & \\
\hline 27:07 & 0.0011 & 0.0021 & 1.0000 & 0.0050 & 0.0100 & 1.0000 & & & & & & & & & & & & \\
\hline $35: 01$ & 0.0032 & 0.0064 & 1.0000 & 0.0050 & 0.0100 & 1.0000 & & & & 0.0050 & 0.0100 & 1.0000 & & & & 0.0071 & 0.0143 & 1.0000 \\
\hline 35:02 & 0.0021 & 0.0043 & 1.0000 & & & & & & & & & & 0.0050 & 0.0100 & 1.0000 & 0.0071 & 0.0143 & 1.0000 \\
\hline 35:03 & 0.0074 & 0.0149 & 1.0000 & 0.0200 & 0.0400 & 1.0000 & & & & 0.0050 & 0.0100 & 1.0000 & 0.0050 & 0.0100 & 1.0000 & 0.0071 & 0.0143 & 1.0000 \\
\hline 35:05 & 0.0191 & 0.0383 & 1.0000 & 0.0300 & 0.0600 & 1.0000 & 0.0200 & 0.0400 & 1.0000 & 0.0200 & 0.0400 & 1.0000 & 0.0100 & 0.0200 & 1.0000 & 0.0143 & 0.0286 & 1.0000 \\
\hline 35:06 & 0.0011 & 0.0021 & 1.0000 & 0.0050 & 0.0100 & 1.0000 & & & & & & & & & & & & \\
\hline $35: 11$ & 0.0011 & 0.0021 & 1.0000 & 0.0050 & 0.0100 & 1.0000 & & & & & & & & & & & & \\
\hline 35:32 & 0.0021 & 0.0043 & 1.0000 & 0.0100 & 0.0200 & 1.0000 & & & & & & & & & & & & \\
\hline $35: 68$ & 0.0011 & 0.0021 & 1.0000 & 0.0050 & 0.0100 & 1.0000 & & & & & & & & & & & & \\
\hline $37: 01$ & 0.0085 & 0.0170 & 1.0000 & 0.0150 & 0.0300 & 1.0000 & & & & 0.0050 & 0.0100 & 1.0000 & 0.0050 & 0.0100 & 1.0000 & 0.0214 & 0.0429 & 1.0000 \\
\hline 38:02 & 0.0426 & 0.0830 & 1.0000 & 0.0400 & 0.0800 & 1.0000 & 0.0550 & 0.1100 & 1.0000 & 0.0300 & 0.0600 & 1.0000 & 0.0500 & 0.0900 & 1.0000 & 0.0357 & 0.0714 & 1.0000 \\
\hline 39:01 & 0.0064 & 0.0128 & 0.5000 & 0.0050 & 0.0100 & 1.0000 & 0.0050 & 0.0100 & 1.0000 & 0.0050 & 0.0100 & 1.0000 & 0.0050 & 0.0100 & 1.0000 & 0.0143 & 0.0286 & 1.0000 \\
\hline 39:06 & 0.0011 & 0.0021 & 1.0000 & 0.0050 & 0.0100 & 1.0000 & & & & & & & & & & & & \\
\hline
\end{tabular}


TABLE 2 | Continued

\begin{tabular}{|c|c|c|c|c|c|c|c|c|c|c|c|c|c|c|c|c|c|c|}
\hline \multirow[t]{2}{*}{ HLA-B } & \multicolumn{3}{|c|}{ Thailand $(n=470)$} & \multicolumn{3}{|c|}{ Southern $(n=100)$} & \multicolumn{3}{|c|}{ Northern $(n=100)$} & \multicolumn{3}{|c|}{ Northeastern $(n=100)$} & \multicolumn{3}{|c|}{ Central $(n=100)$} & \multicolumn{3}{|c|}{ Bangkok $(n=70)$} \\
\hline & AF & CF & $\begin{array}{c}\text { HW } \\
\text { (p-value) }\end{array}$ & AF & CF & $\begin{array}{c}\text { HW } \\
\text { (p-value) }\end{array}$ & AF & CF & $\begin{array}{c}\text { HW } \\
\text { (p-value) }\end{array}$ & AF & CF & $\begin{array}{c}\text { HW } \\
\text { (p-value) }\end{array}$ & AF & CF & $\begin{array}{c}\text { HW } \\
\text { (p-value) }\end{array}$ & AF & CF & $\begin{array}{c}\text { HW } \\
\text { (p-value) }\end{array}$ \\
\hline 39:09 & 0.0160 & 0.0319 & 1.0000 & & & & 0.0150 & 0.0300 & 1.0000 & 0.0300 & 0.0600 & 1.0000 & 0.0250 & 0.0500 & 1.0000 & 0.0071 & 0.0143 & 1.0000 \\
\hline 39:15 & 0.0053 & 0.0106 & 1.0000 & 0.0150 & 0.0300 & 1.0000 & & & & 0.0050 & 0.0100 & 1.0000 & & & & 0.0071 & 0.0143 & 1.0000 \\
\hline 39:49 & 0.0011 & 0.0021 & 1.0000 & & & & & & & & & & 0.0050 & 0.0100 & 1.0000 & & & \\
\hline 40:01 & 0.0660 & 0.1234 & 0.6870 & 0.0300 & 0.0600 & 1.0000 & 0.0750 & 0.1400 & 1.0000 & 0.0950 & 0.1700 & 1.0000 & 0.0650 & 0.1200 & 1.0000 & 0.0643 & 0.1286 & 1.0000 \\
\hline 40:02 & 0.0074 & 0.0149 & 1.0000 & & & & 0.0100 & 0.0200 & 1.0000 & 0.0150 & 0.0300 & 1.0000 & 0.0100 & 0.0200 & 1.0000 & & & \\
\hline 40:06 & 0.0234 & 0.0468 & 1.0000 & 0.0450 & 0.0900 & 1.0000 & 0.0200 & 0.0400 & 1.0000 & 0.0150 & 0.0300 & 1.0000 & 0.0200 & 0.0400 & 1.0000 & 0.0143 & 0.0286 & 1.0000 \\
\hline $40: 11$ & 0.0011 & 0.0021 & 1.0000 & 0.0050 & 0.0100 & 1.0000 & & & & & & & & & & & & \\
\hline 41:01 & 0.0011 & 0.0021 & 1.0000 & 0.0050 & 0.0100 & 1.0000 & & & & & & & & & & & & \\
\hline 41:30 & 0.0011 & 0.0021 & 1.0000 & 0.0050 & 0.0100 & 1.0000 & & & & & & & & & & & & \\
\hline 44:02 & 0.0021 & 0.0043 & 1.0000 & 0.0050 & 0.0100 & 1.0000 & 0.0050 & 0.0100 & 1.0000 & & & & & & & & & \\
\hline 44:03 & 0.0447 & 0.0894 & 1.0000 & 0.0450 & 0.0900 & 1.0000 & 0.0350 & 0.0700 & 1.0000 & 0.0250 & 0.0500 & 1.0000 & 0.0550 & 0.1100 & 1.0000 & 0.0714 & 0.1429 & 1.0000 \\
\hline 46:01 & 0.1404 & 0.2596 & 0.8170 & 0.0650 & 0.1300 & 1.0000 & 0.1850 & 0.3300 & 1.0000 & 0.1300 & 0.2300 & 1.0000 & 0.1900 & 0.3600 & 0.6830 & 0.1286 & 0.2429 & 1.0000 \\
\hline 46:02 & 0.0021 & 0.0043 & 1.0000 & & & & & & & 0.0050 & 0.0100 & 1.0000 & & & & 0.0071 & 0.0143 & 1.0000 \\
\hline 48:01 & 0.0043 & 0.0085 & 1.0000 & 0.0050 & 0.0100 & 1.0000 & 0.0050 & 0.0100 & 1.0000 & 0.0050 & 0.0100 & 1.0000 & 0.0050 & 0.0100 & 1.0000 & & & \\
\hline 48:03 & 0.0106 & 0.0213 & 1.0000 & & & & 0.0050 & 0.0100 & 1.0000 & 0.0050 & 0.0100 & 1.0000 & 0.0250 & 0.0500 & 1.0000 & 0.0214 & 0.0429 & 1.0000 \\
\hline $51: 01$ & 0.0426 & 0.0851 & 1.0000 & 0.0550 & 0.1100 & 1.0000 & 0.0500 & 0.1000 & 1.0000 & 0.0350 & 0.0700 & 1.0000 & 0.0350 & 0.0700 & 1.0000 & 0.0357 & 0.0714 & 1.0000 \\
\hline 51:02 & 0.0138 & 0.0277 & 1.0000 & 0.0250 & 0.0500 & 1.0000 & 0.0100 & 0.0200 & 1.0000 & 0.0100 & 0.0200 & 1.0000 & 0.0100 & 0.0200 & 1.0000 & 0.0143 & 0.0286 & 1.0000 \\
\hline 52:01 & 0.0330 & 0.0638 & 1.0000 & 0.0450 & 0.0800 & 1.0000 & 0.0350 & 0.0700 & 1.0000 & 0.0200 & 0.0400 & 1.0000 & 0.0250 & 0.0500 & 1.0000 & 0.0429 & 0.0857 & 1.0000 \\
\hline 53:01 & 0.0011 & 0.0021 & 1.0000 & & & & 0.0050 & 0.0100 & 1.0000 & & & & & & & & & \\
\hline 54:01 & 0.0085 & 0.0170 & 1.0000 & & & & 0.0100 & 0.0200 & 1.0000 & 0.0100 & 0.0200 & 1.0000 & 0.0150 & 0.0300 & 1.0000 & 0.0071 & 0.0143 & 1.0000 \\
\hline $55: 01$ & 0.0011 & 0.0021 & 1.0000 & & & & & & & 0.0050 & 0.0100 & 1.0000 & & & & & & \\
\hline $55: 02$ & 0.0213 & 0.0426 & 1.0000 & 0.0100 & 0.0200 & 1.0000 & 0.0300 & 0.0600 & 1.0000 & 0.0200 & 0.0400 & 1.0000 & 0.0150 & 0.0300 & 1.0000 & 0.0357 & 0.0714 & 1.0000 \\
\hline $56: 01$ & 0.0106 & 0.0191 & 1.0000 & 0.0100 & 0.0200 & 1.0000 & 0.0100 & 0.0200 & 1.0000 & 0.0150 & 0.0200 & 1.0000 & 0.0100 & 0.0200 & 1.0000 & 0.0071 & 0.0143 & 1.0000 \\
\hline 56:02 & 0.0011 & 0.0021 & 1.0000 & & & & & & & & & & & & & 0.0071 & 0.0143 & 1.0000 \\
\hline 56:04 & 0.0128 & 0.0255 & 1.0000 & 0.0050 & 0.0100 & 1.0000 & 0.0050 & 0.0100 & 1.0000 & 0.0200 & 0.0400 & 1.0000 & 0.0250 & 0.0500 & 1.0000 & 0.0071 & 0.0143 & 1.0000 \\
\hline 57:01 & 0.0117 & 0.0234 & 1.0000 & 0.0100 & 0.0200 & 1.0000 & 0.0100 & 0.0200 & 1.0000 & 0.0100 & 0.0200 & 1.0000 & 0.0150 & 0.0300 & 1.0000 & 0.0143 & 0.0286 & 1.0000 \\
\hline 57:02 & 0.0011 & 0.0021 & 1.0000 & & & & & & & & & & & & & 0.0071 & 0.0143 & 1.0000 \\
\hline $58: 01$ & 0.0638 & 0.1213 & 1.0000 & 0.0600 & 0.1100 & 1.0000 & 0.0500 & 0.1000 & 1.0000 & 0.0800 & 0.1400 & 0.4980 & 0.0750 & 0.1500 & 1.0000 & 0.0500 & 0.1000 & 1.0000 \\
\hline $58: 51$ & 0.0011 & 0.0021 & 1.0000 & 0.0050 & 0.0100 & 1.0000 & & & & & & & & & & & & \\
\hline
\end{tabular}

HLA-B, human leukocyte antigen-B; AF, allele frequency; $C F$, carrier frequency; HW, Hardy-Weinberg equilibrium; Significantly different $p$-value $<0.05$. 
TABLE 3 | The frequency of HLA class I alleles in Thai population and five regions $(n=470)$.

\begin{tabular}{|c|c|c|c|c|c|c|c|c|c|c|c|c|c|c|c|c|c|c|}
\hline \multirow[t]{2}{*}{ HLA-C } & \multicolumn{3}{|c|}{ Thailand $(n=470)$} & \multicolumn{3}{|c|}{ Southern $(n=100)$} & \multicolumn{3}{|c|}{ Northern $(n=100)$} & \multicolumn{3}{|c|}{ Northeastern $(n=100)$} & \multicolumn{3}{|c|}{ Central $(n=100)$} & \multicolumn{3}{|c|}{ Bangkok $(n=70)$} \\
\hline & AF & CF & $\begin{array}{c}\text { HW } \\
\text { (p-value) }\end{array}$ & AF & CF & $\begin{array}{c}\text { HW } \\
\text { (p-value) }\end{array}$ & AF & CF & $\begin{array}{c}\text { HW } \\
\text { (p-value) }\end{array}$ & AF & CF & $\begin{array}{c}\text { HW } \\
\text { (p-value) }\end{array}$ & AF & CF & $\begin{array}{c}\text { HW } \\
\text { (p-value) }\end{array}$ & AF & CF & $\begin{array}{c}\text { HW } \\
\text { (p-value) }\end{array}$ \\
\hline 01:02 & 1713 & 0.3043 & 0.5895 & 0.0750 & 0.1400 & 1.0000 & 0.2200 & 0.3800 & 0.7560 & 0.1700 & 0.3100 & 1.0000 & 0.2200 & 0.3900 & 1.0000 & 0.1714 & 0.3000 & 1.0000 \\
\hline 01:08 & 0011 & 0.0021 & 1.0000 & 0.0050 & 0.0100 & 1.0000 & & & & & & & & & & & & \\
\hline 02:02 & 0.0011 & 0.0021 & 1.0000 & & & & 0.0050 & 0.0100 & 1.0000 & & & & & & & & & \\
\hline 03:02 & 0777 & 0.1468 & 1.0000 & 0.0750 & 0.1300 & 1.0000 & 0.0600 & 0.1200 & 1.0000 & 0.1000 & 0.1800 & 1.0000 & 0.0900 & 0.1800 & 1.0000 & 0.0571 & 0.1143 & 1.0000 \\
\hline 03:03 & .0202 & 0.0362 & 0.4995 & 0.0200 & 0.0400 & 1.0000 & 0.0200 & 0.0400 & 1.0000 & 0.0250 & 0.0400 & 1.0000 & 0.0050 & 0.0100 & 1.0000 & 0.0357 & 0.0571 & 1.0000 \\
\hline 03:04 & .0809 & 0.1404 & 0.0901 & 0.0450 & 0.0900 & 1.0000 & 0.1600 & 0.2500 & 0.3311 & 0.0800 & 0.1400 & 1.0000 & 0.0800 & 0.1500 & 1.0000 & 0.0214 & 0.0429 & 1.0000 \\
\hline 03:08 & .0021 & 0.0043 & 1.0000 & & & & 0.0050 & 0.0100 & 1.0000 & & & & 0.0050 & 0.0100 & 1.0000 & & & \\
\hline 03:09 & 0.0011 & 0.0021 & 1.0000 & & & & 0.0050 & 0.0100 & 1.0000 & & & & & & & & & \\
\hline 03:17 & 0.0021 & 0.0043 & 1.0000 & & & & & & & 0.0050 & 0.0100 & 1.0000 & & & & 0.0071 & 0.0143 & 1.0000 \\
\hline 03:94 & 0.0011 & 0.0021 & 1.0000 & & & & & & & & & & 0.0050 & 0.0100 & 1.0000 & & & \\
\hline 04:01 & 0.0468 & 0.0936 & 1.0000 & 0.0800 & 0.1600 & 1.0000 & 0.0300 & 0.0600 & 1.0000 & 0.0250 & 0.0500 & 1.0000 & 0.0300 & 0.0600 & 1.0000 & 0.0786 & 0.1571 & 1.0000 \\
\hline 04:03 & 0.0426 & 0.0809 & 1.0000 & 0.0400 & 0.0800 & 1.0000 & 0.0450 & 0.0900 & 1.0000 & 0.0600 & 0.1100 & 1.0000 & 0.0200 & 0.0400 & 1.0000 & 0.0500 & 0.0857 & 1.0000 \\
\hline 04:06 & 0.0117 & 0.0234 & 1.0000 & 0.0050 & 0.0100 & 1.0000 & 0.0050 & 0.0100 & 1.0000 & 0.0250 & 0.0500 & 1.0000 & 0.0200 & 0.0400 & 1.0000 & & & \\
\hline 05:01 & .0032 & 0.0064 & 1.0000 & 0.0050 & 0.0100 & 1.0000 & 0.0050 & 0.0100 & 1.0000 & & & & 0.0050 & 0.0100 & 1.0000 & & & \\
\hline 06:02 & 0.0426 & 0.0851 & 1.0000 & 0.0600 & 0.1200 & 1.0000 & 0.0200 & 0.0400 & 1.0000 & 0.0400 & 0.0800 & 1.0000 & 0.0350 & 0.0700 & 1.0000 & 0.0643 & 0.1286 & 1.0000 \\
\hline $06: 89$ & 0.0011 & 0.0021 & 1.0000 & & & & 0.0050 & 0.0100 & 1.0000 & & & & & & & & & \\
\hline 07:01 & 0.0638 & 0.1234 & 1.00 & 0.0700 & 0.1200 & 1. & 0.0400 & 0.0800 & & 0 & 0 & $1 . C$ & 0 & Do & & 0 & Do & 1.0000 \\
\hline 07:02 & 0.1191 & 0.2149 & 0.4752 & 0.0900 & 0.1600 & 1.0000 & 0.1000 & 0.1800 & 1.0000 & 0.1450 & 0.2600 & 1.0000 & 0.1400 & 0.2700 & 1.0000 & 0.1214 & 0.2000 & 0.6195 \\
\hline 07:04 & 0.0500 & 0.0979 & 1.0000 & 0.0650 & 0.1300 & 1.0000 & 0.0300 & 0.0600 & 1.0000 & 0.0650 & 0.1200 & 1.0000 & 0.0350 & 0.0700 & 1.0000 & 0.0571 & 0.1143 & 1.0000 \\
\hline $07: 27$ & 0.0117 & 0.0234 & 1.0000 & 0.0150 & 0.0300 & 1.0000 & 0.0200 & 0.0400 & 1.0000 & & & & 0.0150 & 0.0300 & 1.0000 & 0.0071 & 0.0143 & 1.0000 \\
\hline 07:29 & 0.0085 & 0.0170 & 1.0000 & & & & 0.0050 & 0.0100 & 1.0000 & 0.0200 & 0.0400 & 1.0000 & 0.0100 & 0.0200 & 1.0000 & 0.0071 & 0.0143 & 1.0000 \\
\hline 08:01 & 0.1032 & 0.1915 & 0.7714 & 0.1450 & 0.2700 & 1.0000 & 0.0850 & 0.1700 & 1.0000 & 0.0850 & 0.1500 & 1.0000 & 0.1150 & 0.2200 & 1.0000 & 0.0786 & 0.1286 & 0.4964 \\
\hline 08:02 & 0.0021 & 0.0043 & 1.0000 & 0.0050 & 0.0100 & 1.0000 & & & & & & & & & & 0.0071 & 0.0143 & 1.0000 \\
\hline 08:03 & 0.0032 & 0.00 & 1.0000 & & & & 0.0050 & 0.0100 & 1.0000 & 0.0100 & 0.0200 & U & & & & & & \\
\hline 12:02 & 0.0404 & 0.0787 & 1.0000 & 0.0550 & 0.1000 & 1.0000 & 0.0700 & 0.1400 & 1.0000 & 0.0150 & 0.0300 & 1.0000 & 0.0200 & 0.0400 & 1.0000 & 0.0429 & 0.0857 & 1.0000 \\
\hline $12: 03$ & 0.0138 & 0.0277 & 1.0000 & 0.0200 & 0.0400 & 1.0000 & 0.0100 & 0.0200 & 1.0000 & 0.0200 & 0.0400 & 1.0000 & 0.0050 & 0.0100 & 1.0000 & 0.0143 & 0.0286 & 1.0000 \\
\hline $12: 04$ & 0.0011 & 0.0021 & 1.0000 & & & & & & & 0.0050 & 0.0100 & 1.0000 & & & & & & \\
\hline 14:02 & 0.0287 & 0.0574 & 1.0000 & 0.0350 & 0.0700 & 1.0000 & 0.0300 & 0.0600 & 1.0 & 0.0300 & 0.0600 & 1.0000 & 0.0250 & 0.0500 & 1.0 & 0.0214 & 0.0429 & 1.0000 \\
\hline 15:02 & 0.0340 & 0.0681 & 1.0000 & 0.0600 & 0.1200 & 1.0000 & 0.0150 & 0.0300 & 1.0000 & 0.0250 & 0.0500 & 1.0000 & 0.0300 & 0.0600 & 1.0000 & 0.0429 & 0.0857 & 1.0000 \\
\hline 15:05 & 0.0043 & 0.0085 & 1.0000 & & & & 0.0050 & 0.0100 & 1.0000 & 0.0150 & 0.0300 & 1.0000 & & & & & & \\
\hline 16:02 & 0.0064 & 0.0128 & 1.0000 & 0.0200 & 0.0400 & 1.0000 & & & & & & & 0.0050 & 0.0100 & 1.0000 & 0.0071 & 0.0143 & 1.0000 \\
\hline $17: 01$ & 0.0021 & 0.0043 & 1.0000 & 0.0100 & 0.0200 & 1.0000 & & & & & & & & & & & & \\
\hline 18:01 & 0.0011 & 0.0021 & 1.0000 & & & & & & & & & & & & & 0.0071 & 0.0143 & 1.0000 \\
\hline
\end{tabular}

HLA-C, human leukocyte antigen-C; AF, allele frequency; CF, carrier frequency; HW, Hardy-Weinberg equilibrium; Significantly different p-value $<0.05$. 
TABLE 4 | The frequency of HLA class II alleles in Thai population and five regions $(n=470)$.

\begin{tabular}{|c|c|c|c|c|c|c|c|c|c|c|c|c|c|c|c|c|c|c|}
\hline \multirow[t]{2}{*}{ HLA-DRB1 } & \multicolumn{3}{|c|}{ Thailand $(n=470)$} & \multicolumn{3}{|c|}{ Southern $(n=100)$} & \multicolumn{3}{|c|}{ Northern $(n=100)$} & \multicolumn{3}{|c|}{ Northeastern $(n=100)$} & \multicolumn{3}{|c|}{ Central $(n=100)$} & \multicolumn{3}{|c|}{ Bangkok $(n=70)$} \\
\hline & AF & CF & $\begin{array}{c}\text { HW } \\
\text { (p-value) }\end{array}$ & $\mathrm{AF}$ & CF & $\begin{array}{c}\text { HW } \\
\text { (p-value) }\end{array}$ & AF & CF & $\begin{array}{c}\text { HW } \\
\text { (p-value) }\end{array}$ & AF & CF & $\begin{array}{c}\mathrm{HW} \\
\text { (p-value) }\end{array}$ & AF & CF & $\begin{array}{c}\text { HW } \\
\text { (p-value) }\end{array}$ & AF & CF & $\begin{array}{c}\text { HW } \\
\text { (p-value) }\end{array}$ \\
\hline 01:01 & 0.0032 & 0.0064 & 1.0000 & 0.0050 & 0.0100 & 1.0000 & & & & & & & 0.0100 & 0.0200 & 1.0000 & & & \\
\hline 01:02 & 0.0011 & 0.0021 & 1.0000 & & & & & & & & & & & & & 0.0071 & 0.0143 & 1.0000 \\
\hline 03:01 & 0.0500 & 0.0915 & 0.3737 & 0.0500 & 0.0900 & 1.0000 & 0.0400 & 0.0800 & 1.0000 & 0.0650 & 0.1100 & 1.0000 & 0.0500 & 0.1000 & 1.0000 & 0.0429 & 0.0714 & 1.0000 \\
\hline 03:20 & 0.0032 & 0.0064 & 1.0000 & & & & 0.0050 & 0.0100 & 1.0000 & & & & & & & 0.0143 & 0.0286 & 1.0000 \\
\hline 04:01 & 0.0032 & 0.0064 & 1.0000 & 0.0050 & 0.0100 & 1.0000 & 0.0050 & 0.0100 & 1.0000 & & & & & & & 0.0071 & 0.0143 & 1.0000 \\
\hline 04:02 & 0.0011 & 0.0021 & 1.0000 & 0.0050 & 0.0100 & 1.0000 & & & & & & & & & & & & \\
\hline 04:03 & 0.0234 & 0.0447 & 1.0000 & 0.0300 & 0.0600 & 1.0000 & 0.0150 & 0.0300 & 1.0000 & 0.0050 & 0.0100 & 1.0000 & 0.0250 & 0.0500 & 1.0000 & 0.0500 & 0.0857 & 1.0000 \\
\hline 04:04 & 0.0032 & 0.0064 & 1.0000 & 0.0150 & 0.0300 & 1.0000 & & & & & & & & & & & & \\
\hline 04:05 & 0.0489 & 0.0957 & 1.0000 & 0.0150 & 0.0300 & 1.0000 & 0.0500 & 0.1000 & 1.0000 & 0.0850 & 0.1600 & 1.0000 & 0.0650 & 0.1300 & 1.0000 & 0.0214 & 0.0429 & 1.0000 \\
\hline 04:06 & 0.0032 & 0.0064 & 1.0000 & 0.0050 & 0.0100 & 1.0000 & & & & & & & 0.0050 & 0.0100 & 1.0000 & 0.0071 & 0.0143 & 1.0000 \\
\hline 05:01 & 0.0011 & 0.0021 & 1.0000 & 0.0050 & 0.0100 & 1.0000 & & & & & & & & & & & & \\
\hline 07:01 & 0.0894 & 0.1766 & 0.3737 & 0.0850 & 0.1600 & 1.0000 & 0.0600 & 0.1200 & 1.0000 & 0.0750 & 0.1500 & 1.0000 & 0.1100 & 0.2200 & 1.0000 & 0.1286 & 0.2571 & 1.0000 \\
\hline 08:01 & 0.0011 & 0.0021 & 1.0000 & 0.0050 & 0.0100 & 1.0000 & & & & & & & & & & & & \\
\hline 08:02 & 0.0021 & 0.0043 & 1.0000 & 0.0050 & 0.0100 & 1.0000 & & & & & & & & & & 0.0071 & 0.0143 & 1.0000 \\
\hline 08:03 & 0.0160 & 0.0298 & 1.0000 & 0.0300 & 0.0600 & 1.0000 & 0.0050 & 0.0100 & 1.0000 & 0.0150 & 0.0300 & 1.0000 & 0.0050 & 0.0100 & 1.0000 & 0.0286 & 0.0429 & 1.0000 \\
\hline 08:12 & 0.0021 & 0.0043 & 1.0000 & 0.0050 & 0.0100 & 1.0000 & & & & & & & 0.0050 & 0.0100 & 1.0000 & & & \\
\hline 08:19 & 0.0011 & 0.0021 & 1.0000 & & & & & & & & & & 0.0050 & 0.0100 & 1.0000 & & & \\
\hline 09:01 & 0.0989 & 0.1872 & 1.0000 & 0.0800 & 0.1600 & 1.0000 & 0.1350 & 0.2600 & 1.0000 & 0.1100 & 0.2200 & 1.0000 & 0.0650 & 0.1200 & 1.0000 & 0.1071 & 0.1714 & 0.6195 \\
\hline 10:01 & 0.0213 & 0.0426 & 1.0000 & 0.0300 & 0.0600 & 1.0000 & 0.0200 & 0.0400 & 1.0000 & 0.0200 & 0.0400 & 1.0000 & 0.0150 & 0.0300 & 1.0000 & 0.0214 & 0.0429 & 1.0000 \\
\hline 11:01 & 0.0160 & 0.0319 & 1.0000 & 0.0200 & 0.0400 & 1.0000 & 0.0100 & 0.0200 & 1.0000 & 0.0150 & 0.0300 & 1.0000 & 0.0200 & 0.0400 & 1.0000 & 0.0143 & 0.0286 & 1.0000 \\
\hline 11:02 & 0.0011 & 0.0021 & 1.0000 & 0.0050 & 0.0100 & 1.0000 & & & & & & & & & & & & \\
\hline $11: 04$ & 0.0021 & 0.0043 & 1.0000 & 0.0100 & 0.0200 & 1.0000 & & & & & & & & & & & & \\
\hline 11:05 & 0.0021 & 0.0043 & 1.0000 & 0.0050 & 0.0100 & 1.0000 & & & & & & & 0.0050 & 0.0100 & 1.0000 & & & \\
\hline 11:06 & 0.0138 & 0.0277 & 1.0000 & 0.0100 & 0.0200 & 1.0000 & & & & 0.0200 & 0.0400 & 1.0000 & 0.0250 & 0.0500 & 1.0000 & 0.0143 & 0.0286 & 1.0000 \\
\hline 12:01 & 0.0074 & 0.0149 & 1.0000 & 0.0050 & 0.0100 & 1.0000 & 0.0100 & 0.0200 & 1.0000 & & & & 0.0150 & 0.0300 & 1.0000 & 0.0071 & 0.0143 & 1.0000 \\
\hline 12:02 & 0.1532 & 0.2851 & 0.8253 & 0.1300 & 0.2500 & 1.0000 & 0.1350 & 0.2600 & 1.0000 & 0.1600 & 0.3100 & 0.6212 & 0.1650 & 0.2900 & 1.0000 & 0.1857 & 0.3286 & 1.0000 \\
\hline 12:07 & 0.0032 & 0.0064 & 1.0000 & & & & 0.0050 & 0.0100 & 1.0000 & 0.0050 & 0.0100 & 1.0000 & & & & 0.0071 & 0.0143 & 1.0000 \\
\hline 12:12 & 0.0011 & 0.0021 & 1.0000 & & & & 0.0050 & 0.0100 & 1.0000 & & & & & & & & & \\
\hline 12:16 & 0.0021 & 0.0043 & 1.0000 & 0.0050 & 0.0100 & 1.0000 & & & & & & & & & & 0.0071 & 0.0143 & 1.0000 \\
\hline 13:01 & 0.0096 & 0.0191 & 1.0000 & 0.0200 & 0.0400 & 1.0000 & & & & 0.0050 & 0.0100 & 1.0000 & 0.0050 & 0.0100 & 1.0000 & 0.0214 & 0.0429 & 1.0000 \\
\hline 13:02 & 0.0138 & 0.0277 & 1.0000 & 0.0300 & 0.0600 & 1.0000 & & & & 0.0150 & 0.0300 & 1.0000 & 0.0100 & 0.0200 & 1.0000 & 0.0143 & 0.0286 & 1.0000 \\
\hline 13:12 & 0.0074 & 0.0149 & 1.0000 & & & & 0.0050 & 0.0100 & 1.0000 & 0.0150 & 0.0300 & 1.0000 & & & & 0.0214 & 0.0429 & 1.0000 \\
\hline 14:01 & 0.0596 & 0.1085 & 1.0000 & 0.0400 & 0.0700 & 1.0000 & 0.1150 & 0.2000 & 0.6195 & 0.0350 & 0.0700 & 1.0000 & 0.0650 & 0.1200 & 1.0000 & 0.0357 & 0.0714 & 1.0000 \\
\hline 14:03 & 0.0011 & 0.0021 & 1.0000 & 0.0050 & 0.0100 & 1.0000 & & & & & & & & & & & & \\
\hline 14:04 & 0.0234 & 0.0447 & 1.0000 & 0.0250 & 0.0500 & 1.0000 & 0.0150 & 0.0300 & 1.0000 & 0.0300 & 0.0500 & 1.0000 & 0.0300 & 0.0600 & 1.0000 & 0.0143 & 0.0286 & 1.0000 \\
\hline 14:05 & 0.0074 & 0.0149 & 1.0000 & & & & 0.0150 & 0.0300 & 1.0000 & 0.0050 & 0.0100 & 1.0000 & & & & 0.0214 & 0.0429 & 1.0000 \\
\hline 14:07 & 0.0011 & 0.0021 & 1.0000 & 0.0050 & 0.0100 & 1.0000 & & & & & & & & & & & & \\
\hline 14:22 & 0.0021 & 0.0043 & 1.0000 & & & & & & & & & & 0.0050 & 0.0100 & 1.0000 & 0.0071 & 0.0143 & 1.0000 \\
\hline 15:01 & 0.0809 & 0.1532 & 1.0000 & 0.1050 & 0.2000 & 1.0000 & 0.0750 & 0.1500 & 1.0000 & 0.0650 & 0.1200 & 1.0000 & 0.0950 & 0.1800 & 1.0000 & 0.0571 & 0.1000 & 1.0000 \\
\hline 15:02 & 0.1447 & 0.2638 & 0.8292 & 0.1600 & 0.3100 & 0.6212 & 0.1650 & 0.2900 & 1.0000 & 0.1700 & 0.3100 & 1.0000 & 0.1250 & 0.2200 & 1.0000 & 0.0857 & 0.1571 & 1.0000 \\
\hline 15:03 & 0.0011 & 0.0021 & 1.0000 & & & & & & & & & & & & & 0.0071 & 0.0143 & 1.0000 \\
\hline $15: 06$ & 0.0011 & 0.0021 & 1.0000 & 0.0050 & 0.0100 & 1.0000 & & & & & & & & & & & & \\
\hline 15:24 & 0.0011 & 0.0021 & 1.0000 & & & & & & & 0.0050 & 0.0100 & 1.0000 & & & & & & \\
\hline
\end{tabular}




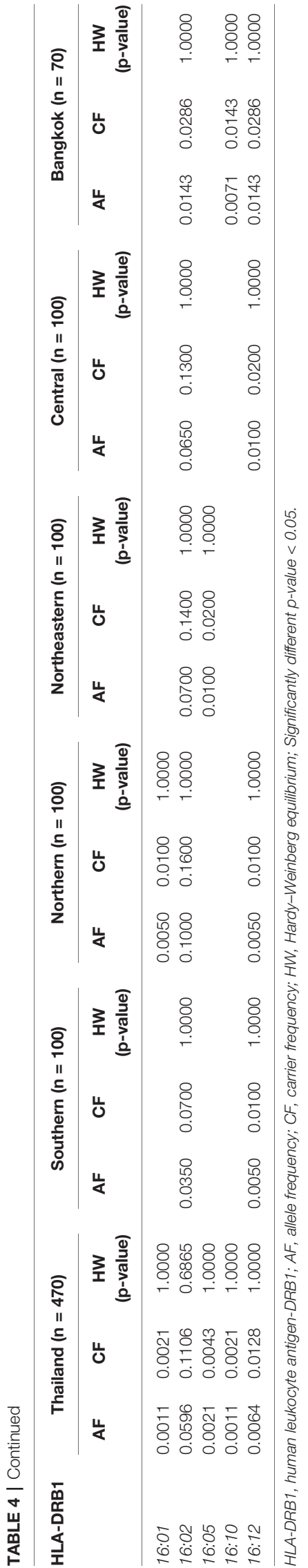

the highest allele in the Southern, Northern, and Central groups. On the contrary, HLA-DQA1*01:02 allele was found more commonly in both Northeastern and Bangkok regions. The frequency distribution of $H L A-D Q B 1$ alleles includes $H L A$ $D Q B 1^{*} 05: 02$ (21.28\%), $-D Q B 1^{*} 03: 01$ (17.23\%), $-D Q B 1^{*} 05: 01$ (14.04\%), $-D Q B 1^{*} 03: 03(11.28 \%),-D Q B 1^{*} 02: 02(7.23 \%)$, $-D Q B 1^{*} 06: 01(7.13 \%)$, and $-D Q B 1^{*} 02: 01(5.43 \%)$, as shown in Table 6. $H L A-D Q B 1{ }^{*} 03: 01$ was the main allele in both Southern and Bangkok regions meanwhile $H L A-D Q B 1^{*} 05: 02$ was found more common in Northern, Northeastern, and Central. For the statistical analysis, the allele frequency of $H L A-A,-B,-C,-D R B 1$, $-D Q A 1$, and $-D Q B 1$ were tested from Hardy-Weinberg equilibrium ( $p$-value $<0.05)$. There was no significant differentiation in each $H L A$ class I and class II alleles except only $H L A-D Q A 1^{\star} 03: 02$ allele in Thai population.

\section{Distribution of Pharmacogenetics Markers}

The allele frequencies across Thailand five regions of HLA$B^{\star}$ 15:02 (IMGT/HLA ID: HLA00165) which induces the risk of having SJS/TEN upon taking aromatic antiepileptic drug [CBZ, oxcarbazepine (OXC), phenytoin (PHT), and lamotrigine (LTG)], had no significant differences $(p$-value $>0.05)$ (Table 7). The frequency of $H L A-B^{\star} 15: 02$ allele was much lower in other populations, namely, African Americans (Cao et al., 2001), Caucasians (Cao et al., 2001), Hispanics (Cao et al., 2001), and North Americans (Cao et al., 2001) (Table 8A and Figure 1). Additionally, $H L A-B^{*} 15: 02$ allele belongs to the HLA-B75 family, which consists of $H L A-B^{*} 15: 11$ (IMGT/HLA ID: HLA00174) $(0.50 \%)$ and $H L A-B^{*} 15: 21$ (IMGT/HLA ID: HLA00184) (0.50\%) in Southern group, whereas $H L A-B^{*} 15: 08$ (IMGT/HLA ID: HLA00171) was not found in this population. In terms of HLA-A ${ }^{\star} 31: 01$ (IMGT/HLA ID: HLA00097) allele which associates with CBZ-induced CADRs, neither the frequencies within Thailand nor among different populations were significantly different (Tables $7, \mathbf{8 A}$ ).

HLA- ${ }^{\star} 58: 01$ (IMGT/HLA ID: HLA00386) allele was similarly distributed in Thais (6.38\%), African Americans (6.37\%), and Asians (7.38\%) (Yang et al., 2016) and higher than those reported in Caucasians (Cao et al., 2001), Hispanics (Cao et al., 2001), and North Americans (Cao et al., 2001) (Tables 7, 8A). However, HLA- $B^{\star 58: 01}$ allele was not significantly different across different populations. Furthermore, other HLA alleles which are associated with allopurinol-induced SJS/TEN such as $H L A-A^{*} 33: 03$ (IMGT/ HLA ID: HLA00106), HLA-C*03:02 (IMGT/HLA ID: HLA00410), and HLA-DRB $1^{*} 15: 02$ (IMGT/HLA ID: HLA00867) alleles were found to have higher frequency in Thai population than others. Note that the high distribution of HLA-DRB1*13:02 (IMGT/HLA ID: HLA00798) allele was observed in African Americans (8.5\%) and Japanese (7.7\%) but not so much (1.38\%) among Thais (Tables 8A, B).

HLA- ${ }^{\star} 13: 01$ (IMGT/HLA ID: HLA00152) allele has been reported to be associated with dapsone and salazosulfapyridineinduced drug reaction with eosinophilia and systemic symptoms (DRESS). The frequencies of $H L A-B^{\star} 13: 01$ were similar among Thai population $(p$-value $=0.7450)$ and higher than African Americans, Caucasians, Hispanics, and North American 
TABLE 5 | The frequency of HLA class II alleles in Thai population and five regions $(n=470)$.

\begin{tabular}{|c|c|c|c|c|c|c|c|c|c|c|c|c|c|c|c|c|c|c|}
\hline \multirow[t]{2}{*}{ HLA-DQA1 } & \multicolumn{3}{|c|}{ Thailand $(n=470)$} & \multicolumn{3}{|c|}{ Southern $(n=100)$} & \multicolumn{3}{|c|}{ Northern $(n=100)$} & \multicolumn{3}{|c|}{ Northeastern $(n=100)$} & \multicolumn{3}{|c|}{ Central $(n=100)$} & \multicolumn{3}{|c|}{ Bangkok $(n=70)$} \\
\hline & AF & CF & $\begin{array}{c}\text { HW } \\
\text { (p-value) }\end{array}$ & AF & CF & $\begin{array}{c}\mathrm{HW} \\
\text { (p-value) }\end{array}$ & AF & CF & $\begin{array}{c}\text { HW } \\
\text { (p-value) }\end{array}$ & AF & CF & $\begin{array}{c}\text { HW } \\
\text { (p-value) }\end{array}$ & AF & CF & $\begin{array}{c}\mathrm{HW} \\
\text { (p-value) }\end{array}$ & AF & CF & $\begin{array}{c}\text { HW } \\
\text { (p-value) }\end{array}$ \\
\hline $01: 01$ & 0.2489 & 0.4170 & 0.3105 & 0.2350 & 0.4000 & 0.7740 & 0.3250 & 0.5000 & 0.5280 & 0.2450 & 0.4300 & 1.0000 & 0.2400 & 0.4200 & 1.0000 & 0.1786 & 0.3000 & 0.6810 \\
\hline 01:02 & 0.2223 & 0.3894 & 0.7692 & 0.2150 & 0.3900 & 1.0000 & 0.2150 & 0.3700 & 0.7560 & 0.2600 & 0.4300 & 0.7940 & 0.2200 & 0.3900 & 1.0000 & 1929 & 0.3571 & 1.0000 \\
\hline 01:03 & 0.0383 & 0.0723 & 1.0000 & 0.0850 & 0.1600 & 1.0000 & 0.0150 & 0.0300 & 1.0000 & 0.0150 & 0.0300 & 1.0000 & 0.0350 & 0.0700 & 1.0000 & 0.0429 & 0.0714 & 1.0000 \\
\hline 01:06 & 0.0011 & 0.0021 & 1.0000 & & & & & & & & & & & & & 0.0071 & 0.0143 & 1.0000 \\
\hline 02:01 & 0.0872 & 0.1723 & 0.3740 & 0.0800 & 0.1500 & 1.0000 & 0.0600 & 0.1200 & 1.0000 & 0.0700 & 0.1400 & 1.0000 & 0.1100 & 0.2200 & 1.0000 & 0.1286 & 0.2571 & 1.0000 \\
\hline 03:01 & 0.0457 & 0.0851 & 0.6240 & 0.0650 & 0.1200 & 1.0000 & 0.0350 & 0.0700 & 1.0000 & 0.0300 & 0.0600 & 1.0000 & 0.0400 & 0.0700 & 1.0000 & 0.0643 & 0.1143 & 1.0000 \\
\hline 03:02 & 0.1330 & 0.2660 & 0.0080 & 0.0850 & 0.1700 & 1.0000 & 0.1700 & 0.3400 & 0.2460 & 0.1700 & 0.3400 & 0.2460 & 0.1250 & 0.2500 & 0.4980 & 0.1071 & 0.2143 & 1.0000 \\
\hline 04:01 & 0.0021 & 0.0043 & 1.0000 & & & & 0.0050 & 0.0100 & 1.0000 & & & & & & & 0.0071 & 0.0143 & 1.0000 \\
\hline 05:01 & 0.0543 & 0.1043 & 1.0000 & 0.0600 & 0.1100 & 1.00 & 0.0450 & 0.0900 & 1.0000 & 0.0600 & 0.1100 & 0 & 0.0500 & 0.1000 & 1.0 & 0.0571 & 0.1143 & 1.0000 \\
\hline 05:03 & 0.0117 & 0.0234 & 1.0000 & 0.0050 & 0.0100 & 1.0000 & 0.0050 & 0.0100 & 1.0000 & 0.0150 & 0.0300 & 1.0000 & 0.0150 & 0.0300 & 1.0000 & 0.0214 & 0.0429 & 1.0000 \\
\hline 05:05 & 0.0340 & 0.0660 & 1.0000 & 0.0350 & 0.0600 & 1.0000 & 0.0150 & 0.0300 & 1.0000 & 0.0400 & 0.0800 & 1.0000 & 0.0450 & 0.0900 & 1.0000 & 0.0357 & 0.0714 & 1.0000 \\
\hline 05:08 & 0.0011 & 0.0021 & 1.0000 & & & & 0.0050 & 0.0100 & 1.0000 & & & & & & & & & \\
\hline 06:01 & 0.1202 & 0.2277 & 0.7800 & 0.1350 & 0.2600 & 1.0000 & 0.1050 & 0.2100 & 1.0000 & 0.0950 & 0.1800 & 1.0000 & 0.1200 & 0.2200 & 1.0000 & 0.1571 & 0.2857 & 1.0000 \\
\hline
\end{tabular}

HLA-DQA1, human leukocyte antigen-DQA1; AF, allele frequency; CF, carrier frequency; HW, Hardy-Weinberg equilibrium; Significantly different $p$-value $<0.05$.

TABLE 6 | The frequency of HLA class II alleles in Thai population and five regions $(n=470)$.

\begin{tabular}{|c|c|c|c|c|c|c|c|c|c|c|c|c|c|c|c|c|c|c|}
\hline \multirow[t]{2}{*}{ HLA-DQB1 } & \multicolumn{3}{|c|}{ Thailand $(n=470)$} & \multicolumn{3}{|c|}{ Southern $(n=100)$} & \multicolumn{3}{|c|}{ Northern $(n=100)$} & \multicolumn{3}{|c|}{ Northeastern $(n=100)$} & \multicolumn{3}{|c|}{ Central $(n=100)$} & \multicolumn{3}{|c|}{ Bangkok $(n=70)$} \\
\hline & AF & CF & $\begin{array}{c}\text { HW } \\
\text { (p-value) }\end{array}$ & AF & CF & $\begin{array}{c}\text { HW } \\
\text { (p-value) }\end{array}$ & AF & CF & $\begin{array}{c}\text { HW } \\
\text { (p-value) }\end{array}$ & AF & CF & $\begin{array}{c}\text { HW } \\
\text { (p-value) }\end{array}$ & AF & CF & $\begin{array}{c}\text { HW } \\
\text { (p-value) }\end{array}$ & AF & CF & $\begin{array}{c}\text { HW } \\
\text { (p-value) }\end{array}$ \\
\hline 02:01 & 0.0543 & 0.1000 & 0.3740 & 0.0550 & 0.1000 & 1.0000 & 0.0450 & 0.0900 & 1.0000 & 0.0650 & 0.1100 & 0.4980 & 0.0500 & 0.1000 & 1.0000 & 0.0571 & 0.1000 & 1.0000 \\
\hline 02:02 & 0723 & 0.1447 & 0.5000 & 0.0700 & 0.1400 & 1.0000 & 0.0500 & 0.1000 & 1.0000 & 0.0600 & 0.1200 & 1.0000 & 0.0850 & 0.1700 & 1.0000 & 0.1071 & 0.2143 & 1.0000 \\
\hline 03:01 & 1723 & 0.3213 & 0.6850 & 0.1850 & 0.3300 & 1.0000 & 0.1400 & 0.2700 & 1.0000 & 0.1500 & 0.2900 & 1.0000 & 0.1800 & 0.3400 & 1.0000 & 0.2214 & 0.4000 & 1.0000 \\
\hline 03:02 & 0.0426 & 0.0787 & 0.6240 & 0.0600 & 0.1100 & 1.0000 & 0.0300 & 0.0600 & 1.0000 & 0.0200 & 0.0400 & 1.0000 & 0.0400 & 0.0700 & 1.0000 & 0.0714 & 0.1286 & 1.0000 \\
\hline 03:03 & 0.1128 & 0.2149 & 0.7620 & 0.0850 & 0.1700 & 1.0000 & 0.1350 & 0.2600 & 1.0000 & 0.1250 & 0.2500 & 1.0000 & 0.0950 & 0.1800 & 1.0000 & 0.1286 & 0.2143 & 0.6200 \\
\hline 03:38 & 0.0011 & 0.0021 & 1.0000 & 0.0050 & 0.0100 & 1.0000 & & & & & & & & & & & & \\
\hline 04:01 & 0.0383 & 0.0745 & 1.0000 & 0.0150 & 0.0300 & 1.0000 & 0.0400 & 0.0800 & 1.0000 & 0.0600 & 0.1100 & 1.0000 & 0.0550 & 0.1100 & 1.0000 & 0.0143 & 0.0286 & 1.0000 \\
\hline 04:02 & 0.0032 & 0.0064 & 1.0000 & & & & 0.0050 & 0.0100 & 1.0000 & 0.0050 & 0.0100 & 1.0000 & & & & 0.0071 & 0.0143 & 1.0000 \\
\hline 05:01 & 0.1404 & 0.2574 & 0.8210 & 0.1350 & 0.2600 & 1.0000 & 0.1700 & 0.2900 & 0.7210 & 0.1600 & 0.3000 & 1.0000 & 0.1350 & 0.2500 & 1.0000 & 0.0857 & 0.1571 & 1.0000 \\
\hline 05:02 & 0.2128 & 0.3872 & 0.7440 & 0.1600 & 0.2800 & 1.0000 & 0.2850 & 0.4900 & 1.0000 & 0.2400 & 0.4500 & 0.4980 & 0.2000 & 0.3900 & 0.3690 & 0.1643 & 0.3000 & 1.0000 \\
\hline 05:03 & 0.0404 & 0.0787 & 1.0000 & 0.0450 & 0.0900 & 1.0000 & 0.0400 & 0.0800 & 1.0000 & 0.0350 & 0.0600 & 1.0000 & 0.0500 & 0.1000 & 1.0000 & 0.0286 & 0.0571 & 1.0000 \\
\hline 05:66 & 0.0011 & 0.0021 & 1.0000 & 0.0050 & 0.0100 & 1.0000 & & & & & & & & & & & & \\
\hline 06:01 & 0.0713 & 0.1340 & 0.6870 & 0.1150 & 0.2100 & 1.0000 & 0.0400 & 0.0800 & 1.0000 & 0.0550 & 0.1100 & 1.0000 & 0.0850 & 0.1600 & 1.0000 & 0.0571 & 0.1000 & 1.0000 \\
\hline 06:02 & 0.0149 & 0.0298 & 1.0000 & 0.0150 & 0.0300 & 1.0000 & 0.0150 & 0.0300 & 1.0000 & 0.0100 & 0.0200 & 1.0000 & 0.0100 & 0.0200 & 1.0000 & 0.0286 & 0.0571 & 1.0000 \\
\hline 06:03 & 0.0074 & 0.0149 & 1.0000 & 0.0200 & 0.0400 & 1.0000 & & & & & & & 0.0050 & 0.0100 & 1.0000 & 0.0143 & 0.0286 & 1.0000 \\
\hline 06:04 & 0.0053 & 0.0106 & 1.0000 & 0.0100 & 0.0200 & 1.0000 & & & & 0.0050 & 0.0100 & 1.0000 & 0.0050 & 0.0100 & 1.0000 & 0.0071 & 0.0143 & 1.0000 \\
\hline 06:05 & 0.0043 & 0.0085 & 1.0000 & 0.0050 & 0.0100 & 1.0000 & & & & 0.0050 & 0.0100 & 1.0000 & 0.0050 & 0.0100 & 1.0000 & 0.0071 & 0.0143 & 1.0000 \\
\hline 06:09 & 0.0043 & 0.0085 & 1.0000 & 0.0150 & 0.0300 & 1.0000 & & & & 0.0050 & 0.0100 & 1.0000 & & & & & & \\
\hline $06: 10$ & 0.0011 & 0.0021 & 1.0000 & & & & 0.0050 & 0.0100 & 1.0000 & & & & & & & & & \\
\hline
\end{tabular}

HLA-DQB1, human leukocyte antigen-DQB1; AF, allele frequency; CF, carrier frequency; HW, Hardy-Weinberg equilibrium; Significantly different $p$-value $<0.05$. 
TABLE 7 | Distribution of pharmacogenetics markers in five regions and Thai population.

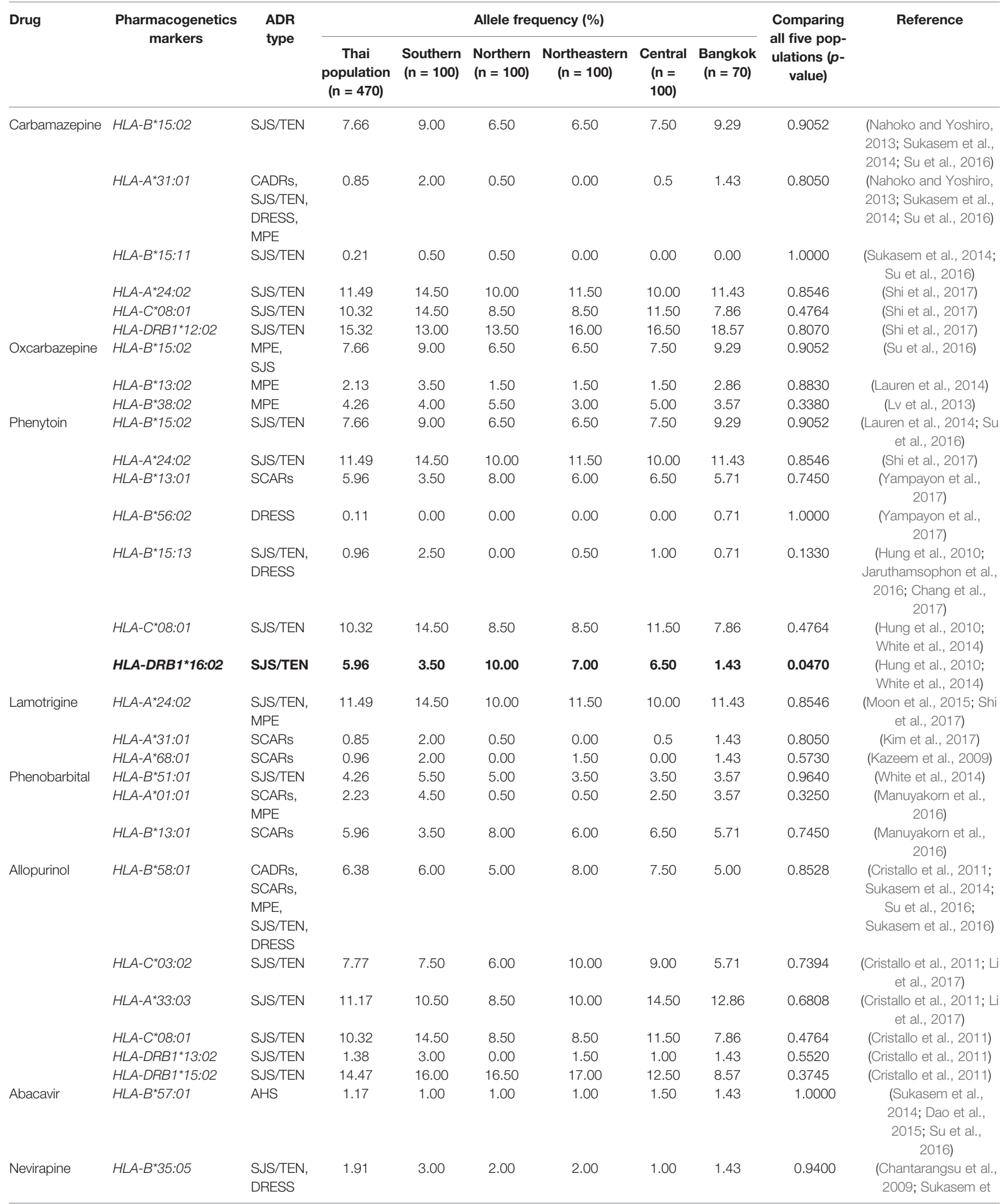

(Continued) 
TABLE 7 | Continued

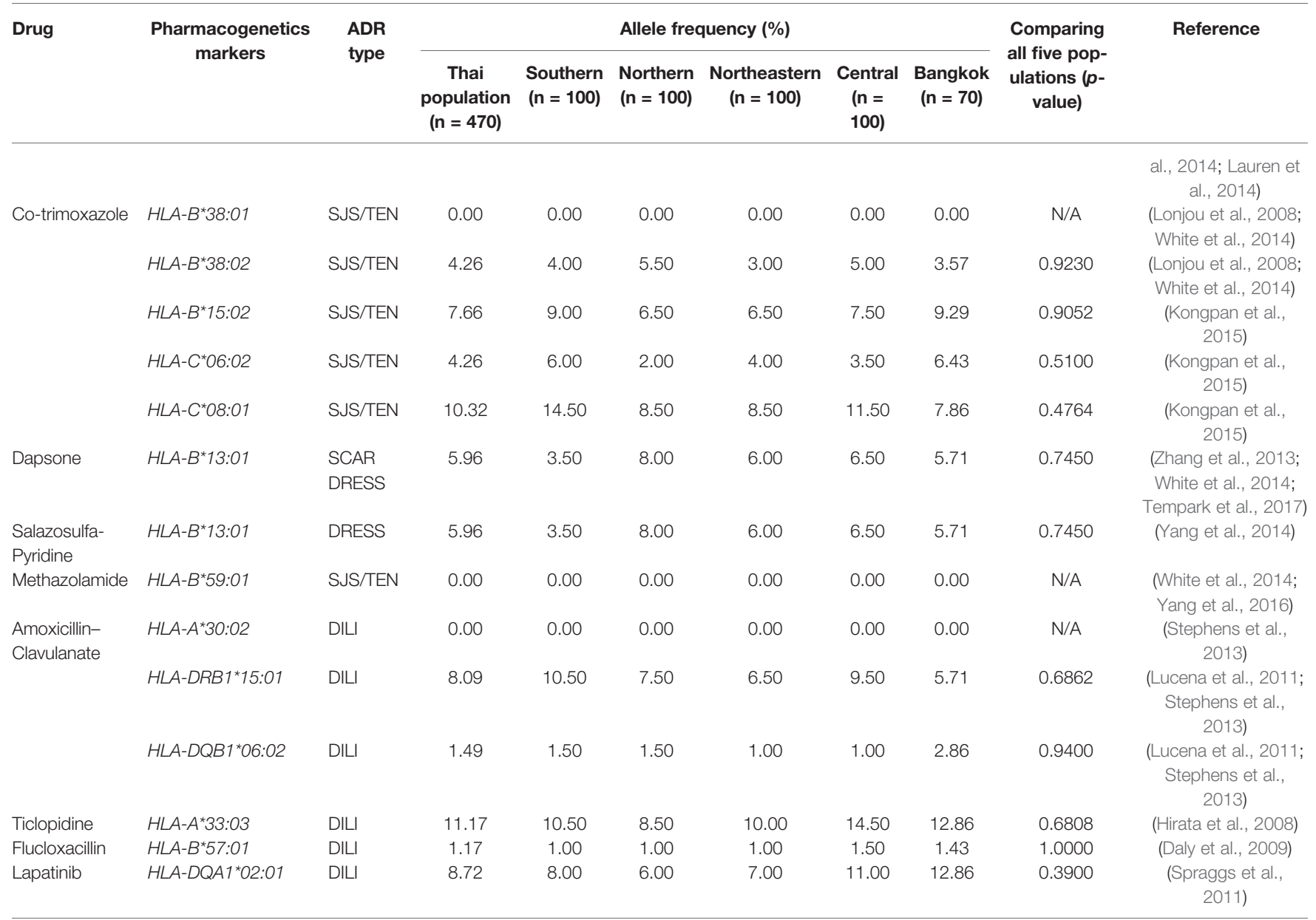

ADR, adverse drug reactions; HLA-A, human leukocyte antigen-A; HLA-B, human leukocyte antigen-B; HLA-C, human leukocyte antigen-C; HLA-DRB1, human leukocyte antigen-DRB1; HLA-DQA1, human leukocyte antigen-DQA1; HLA-DQB1, human leukocyte antigen-DQB1; AHS, abacavir hypersensitivity; CADRs, cutaneous adverse drug reactions; DRESS, drug

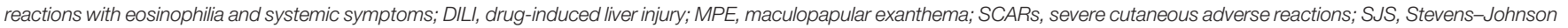
syndrome; TEN, toxic epidermal necrolysis; N/A, not available; Significant difference $p$-value $<0.05$ by Pearson's chi-square test.

Bolded text: Data analysis result was presented statistical significance $(p$-value $<0.05)$.

(Tables 7, 8A). Moreover, co-trimoxazole-induced SJS/TEN associated with $H L A-B^{\star} 15: 02(7.66 \%)$ and $-C^{\star} 08: 01(10.32 \%)$ alleles was higher within Thai population than that of African Americans, Caucasians, Hispanics, and North American. Furthermore, $H L A-A^{\star} 33: 03$ allele which is associated with ticlopidine-induced liver injury appeared to be higher $(11.17 \%)$ than the allele frequencies from Caucasians (Cao et al., 2001), Hispanics (Cao et al., 2001), and North American (Cao et al., 2001). The allele frequencies of $H L A-D Q B 1^{\star} 06: 02$ (IMGT/HLA ID: HLA00646) allele which is associated with amoxicillinclavulanate-induced liver injury were found to have higher frequency in African Americans and Caucasians in contrast to the allele frequency from Thai population (Table 8B).

\section{Population Structure Analysis}

We used PCA to observe potential subgroups within a given 470 Thai individuals. Instead of using only six HLA halplotypes (from both class I and II), we extract 403 polymorphism probes and used them in the analysis. PCA revealed three prominent subpopulations in which samples from
Northeastern (NE:yellow) and Southern (South:pink) groups were somewhat separated (see plots of PC1 vs. PC2 and PC2 vs. PC3 in Figure 2). The third subpopulation-placed in the middle of Northeastern and Southern groups-comprised samples from Central (sky blue), North (green), and Bangkok (BKK:red). The PCA plot between PC1 vs. PC3 did not clearly show subpopulations.

STRUCTURE uses Bayesian to infer/predict a contribution to potential subpopulations $(\mathrm{K})$ from estimated genetic variation frequencies. In our case, $403 \mathrm{HLA}$ polymorphism probe signals were used to represent the genetic variations. Since this work recruited volunteers from five regions based on recent demographic information, we set $\mathrm{K}=10$ to cover these five demographic groups. CLUMPAK reported eight as the best number of genetic groups (subpopulations) for both Evanno's $(\Delta K)$ and Pritchard's (likelihood K). Figure 3 shows patterns of individuals' assignments to $\mathrm{K}$ populations. Each row in this figure shows proportion of individuals' contribution to $\mathrm{K}$ subpopulations, from $K=2$ to $K=9$. In particular, $K$ colors on each vertical bar reveal genetic composition (admixture). 
TABLE 8A | Comparison of pharmacogenetics markers in Thai and other populations.

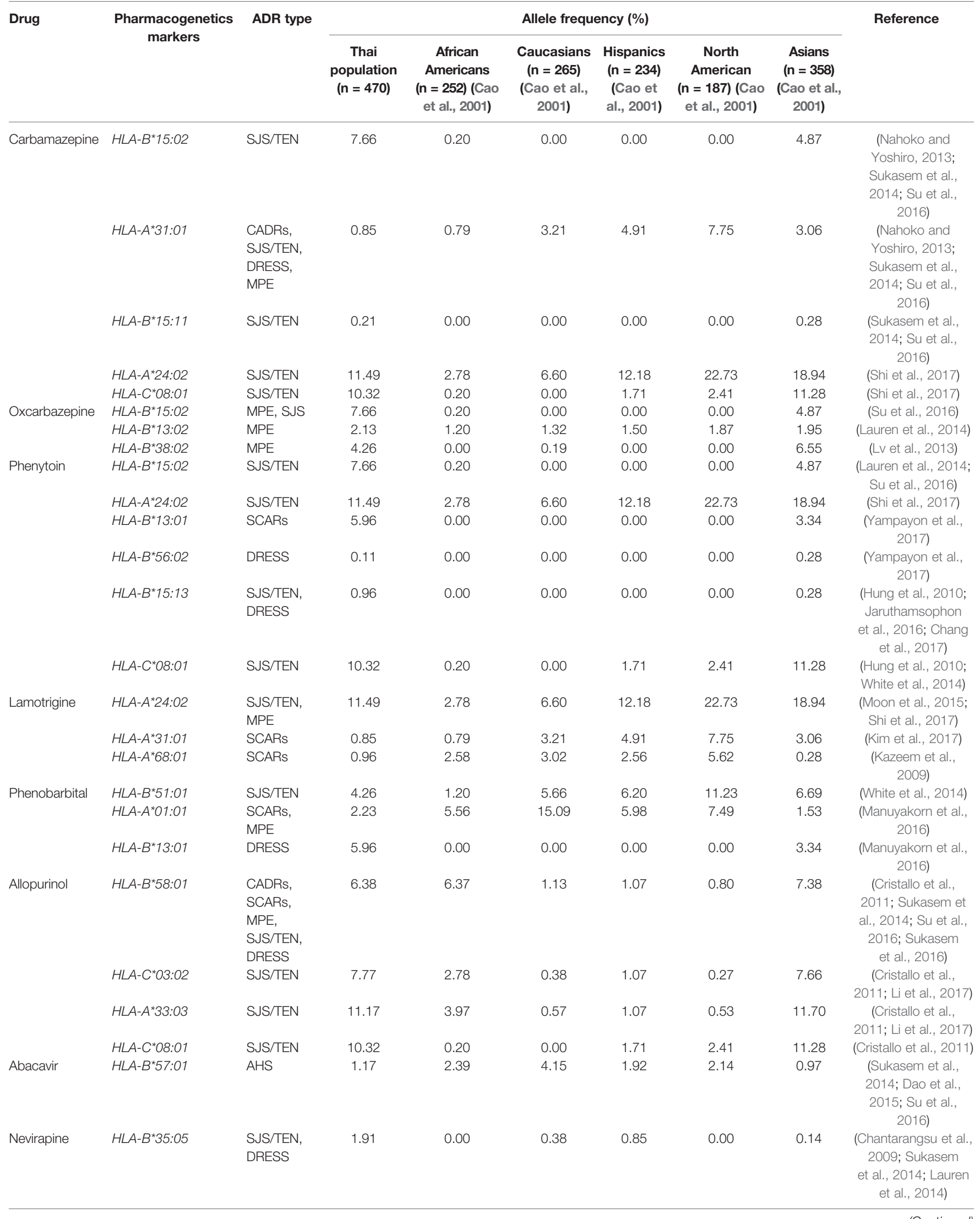

(Continued) 
TABLE 8A | Continued

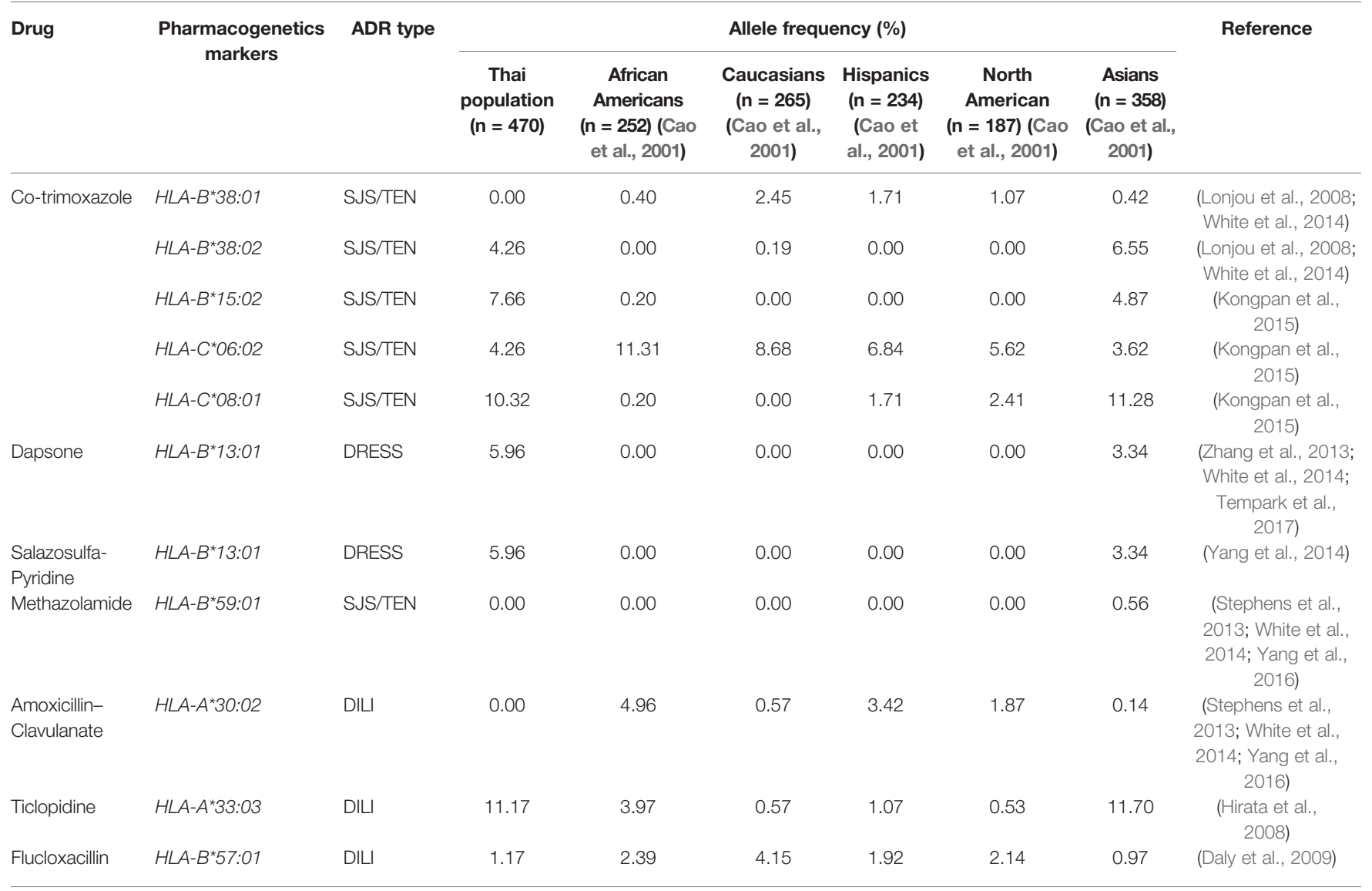

HLA-A, human leukocyte antigen-A; HLA-B, human leukocyte antigen-B; HLA-C, human leukocyte antigen-C; AHS, abacavir hypersensitivity; CADRs, cutaneous adverse drug reactions; DRESS, drug reactions with eosinophilia and systemic symptoms; DILI, drug-induced liver injury; MPE, maculopapular exanthema; SCARs, severe cutaneous adverse reactions; SJS, Stevens-Johnson syndrome; TEN, toxic epidermal necrolysis.

TABLE 8B | Comparison of pharmacogenetics markers in Thai and other populations.

\begin{tabular}{|c|c|c|c|c|c|c|c|c|}
\hline Drug & $\begin{array}{c}\text { Pharmacogenetics } \\
\text { markers }\end{array}$ & $\begin{array}{l}\text { ADR } \\
\text { type }\end{array}$ & \multicolumn{5}{|c|}{ Allele frequency (\%) } & Reference \\
\hline Carbamazepine & $H L A-D R B 1 * 12: 02$ & $\begin{array}{l}\text { SJS/ } \\
\text { TEN }\end{array}$ & 15.32 & 0.40 & 0.00 & 1.50 & 7.16 & (Shi et al., 2017) \\
\hline Phenytoin & HLA-DRB1*16:02 & $\begin{array}{l}\text { SJS/ } \\
\text { TEN }\end{array}$ & 5.96 & 0.00 & 0.00 & 0.90 & 2.61 & $\begin{array}{c}\text { (Hung et al., } \\
\text { 2010; White et al., } \\
\text { 2014) }\end{array}$ \\
\hline \multirow[t]{2}{*}{ Allopurinol } & HLA-DRB1*13:02 & $\begin{array}{l}\text { SJS/ } \\
\text { TEN }\end{array}$ & 1.38 & 8.50 & 3.40 & 7.70 & 3.54 & $\begin{array}{l}\text { (Cristallo et al., } \\
\text { 2011) }\end{array}$ \\
\hline & HLA-DRB1*15:02 & $\begin{array}{l}\text { SJS/ } \\
\text { TEN }\end{array}$ & 14.47 & 0.00 & 0.80 & 10.00 & 2.91 & $\begin{array}{l}\text { (Cristallo et al., } \\
\text { 2011) }\end{array}$ \\
\hline $\begin{array}{l}\text { Amoxicillin- } \\
\text { Clavulanate }\end{array}$ & HLA-DRB1*15:01 & DILI & 8.09 & 16.00 & 15.80 & 8.50 & 12.74 & $\begin{array}{l}\text { (Lucena et al., } \\
\text { 2011; Stephens } \\
\text { et al., 2013) }\end{array}$ \\
\hline Lapatinib & $H L A-D Q A 1 * 02: 01$ & DILI & 8.72 & 9.10 & 13.20 & $\mathrm{~N} / \mathrm{A}$ & 7.28 & $\begin{array}{l}\text { (Spraggs et al., } \\
\text { 2011) }\end{array}$ \\
\hline
\end{tabular}

HLA-DRB1, human leukocyte antigen-DRB1; HLA-DQA1, human leukocyte antigen-DQA1; HLA-DQB1, human leukocyte antigen-DQB1; DILI, drug-induced liver injury; SJS, StevensJohnson syndrome; TEN, toxic epidermal necrolysis; N/A, not available. 


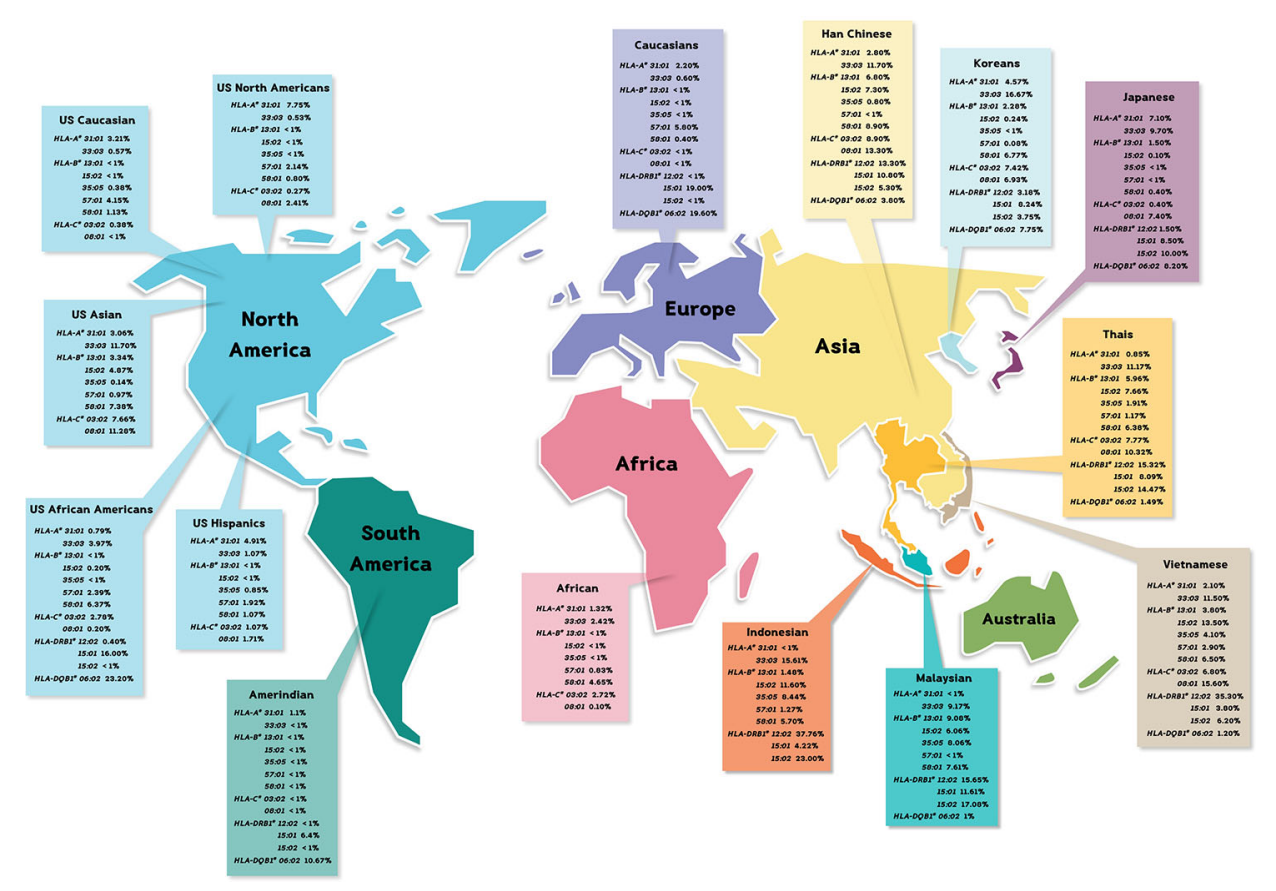

FIGURE 1 | The distribution of pharmacogenetics markers in many populations.

Figure 3 shows the admixture profiles for eight genetic groups ( $\mathrm{K}$ $=8$ ). All 470 individuals are represented by vertical bars, which are grouped according to their reported five geographical origins. Two distinct admixture patterns of Northeastern and Southern regional groups could be observed, while Bangkok and Central regional groups' admixture patterns tend to be similar.

\section{DISCUSSION}

In this study, we examined the frequency distribution of $H L A$ class I and II alleles in five regions of Thailand by HLA-typing of 470 healthy individuals using PCR-SSOs probe. We found the most prevalent alleles of $H L A$ class I and II were $H L A-A^{*} 11: 01$, $-B^{\star} 46: 01,-C^{*} 01: 02,-D R B 1^{\star} 12: 02,-D Q A 1^{\star} 01: 01$, and $-D Q B 1^{*} 05: 02$. When compared with the previous study, the allele frequency of $H L A-B$, our frequency report from 470 healthy cohort was similar to the previous one (Puangpetch et al., 2015). Especially, pharmacogenetic markers associated with different ethnic groups such as $H L A-A^{*} 33: 03,-B^{\star} 15: 02,-B^{\star} 13: 01$, $-C^{\star} 03: 02, \quad-D R B 1^{*} 12: 02, \quad-D R B 1^{\star} 15: 02, \quad-D R B 1^{\star} 16: 02$, and $-D Q B 1^{*} 06: 02$.

Recent studies confirm that SCARs are caused by certain HLA polymorphisms, drugs, peptide, and $\mathrm{T}$ cell (Yun et al., 2012; Iasella et al., 2017). There are four phenotypically distinct SCARs including SJS, TEN, DRESS, or drug-induced hypersensitivity syndrome (DIHS) or hypersensitivity syndrome (HSS), and acute generalized exanthematous pustolosis (AGEP) (Marotti, 2012). SJS and TEN are severe life-threatening reactions and are associated with $\sim 5 \%$ mortality rate, $>30 \%$ SJS and $>30 \%$ TEN (Aihara, 2011). The incidence of SJS/TEN in Southeast Asia (Brunei, Cambodia, Indonesia, Laos, Malaysia, Myanmar, Philippines, Singapore, Vietnam, and Thailand) is largely undetermined. Nevertheless, more and more incidences of culprit drugs have been published, including CBZ (17\%), allopurinol (15\%), beta-lactam antibiotics (13\%), sulfonamide antibiotics (12\%), PHT (9\%), nonsteroidal anti-inflammatory drugs (8\%), LTG (2\%), and phenobarbital (1\%) (Lee et al., 2013). According to the data from the spontaneous reporting of adverse drug reactions during 1984-2014 by Thailand Health Product and Vigilance Center (HPVC), the culprit drugs causing SJS and TEN in Thailand include sulfamethoxazole and trimethoprim, allopurinol, CBZ, nevirapine containing produce, PHT, amoxicillin, phenobarbital, ibuprofen, tetracycline, piroxicam, diclofenac, rifampicin, ceftriaxone, fluconazole, isoniazid, ciprofloxacin, ethambutol, pyrazinamide, amoxicillin and clavulanic acid, and dapsone. (http://thaihpvc.fda.moph.go.th/ thaihvc/Public/News/uploads/hpvc_1_3_4_100538.pdf). DRESS is characterized by fever, cutaneous eruption, internal organ involvement (hepatitis), and hematologic abnormalities (eosinophilia and/or atypical lymphocytosis). The onset of DRESS is approximately $1-8$ weeks after drug initiation with the mortality rate of approximately 10\% (Criado et al., 2012). From 2004 to 2014, the King Chulalongkorn Memorial Hospital Thailand reported list of drugs that could cause DRESS in Thai patients, including PHT, nevirapine, allopurinol, cotrimoxazole, dapsone, $\mathrm{CBZ}$, isoniazid, ciprofloxacin, clindamycin, dacomitinib, danazol, omeprazole, and sulfadiazine (Hiransuthikul et al., 2016). 
A

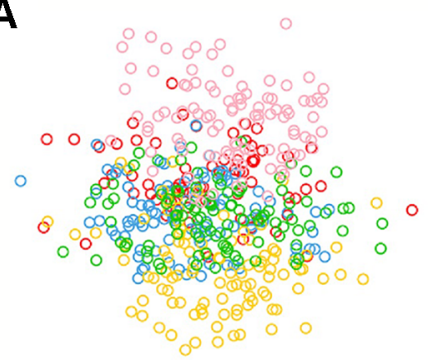

$\tilde{N}_{0}^{\circ}$

PC1

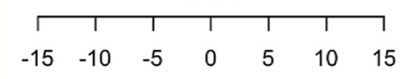

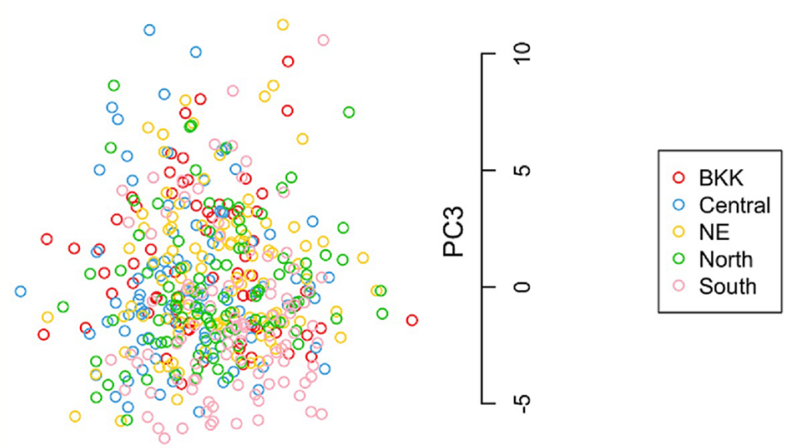

B
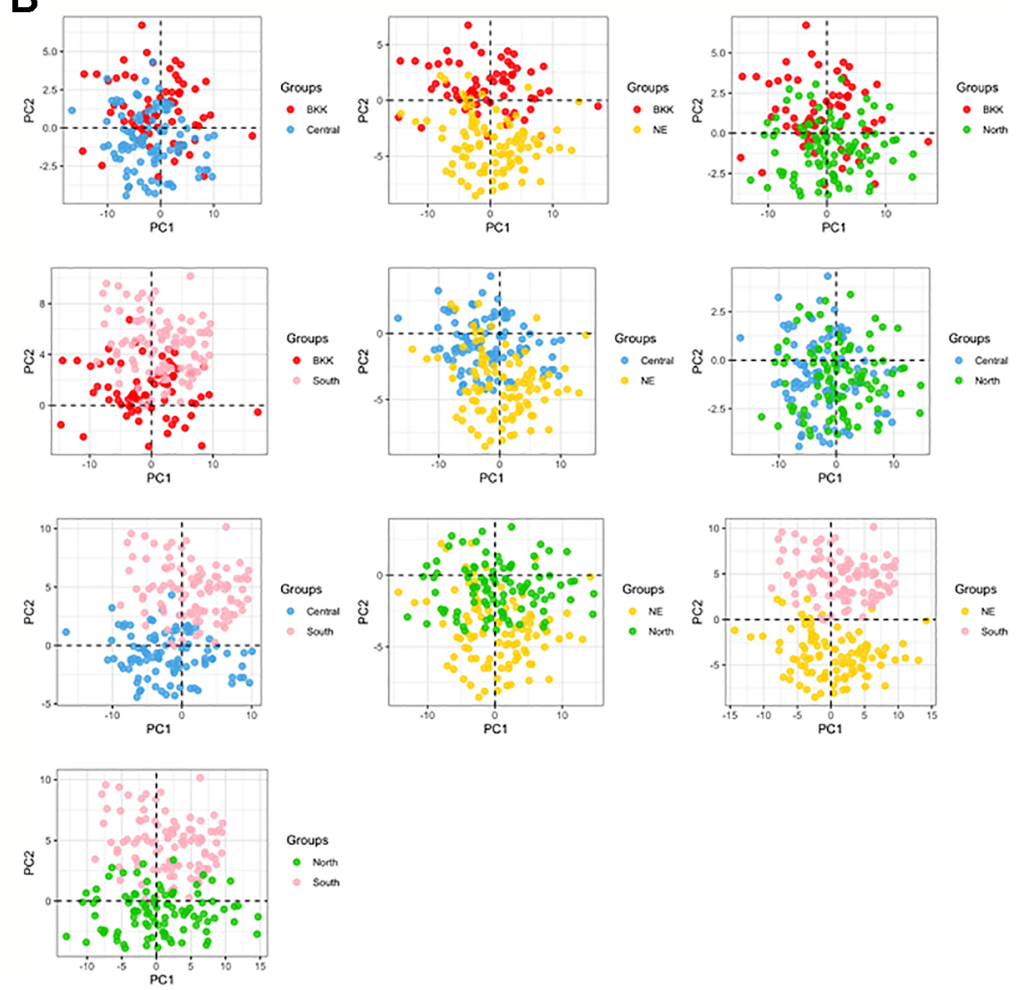

FIGURE 2 | (A) Principal component analysis (PCA) analysis in ggplot library and KRIS library with z-score as the input ffive groups: 100 North, 100 NE (Northeastern), 70 BKK (Bangkok), 100 Central, and 100 South]. (B) All compare between each population by Principal Component Analysis (PCA). 


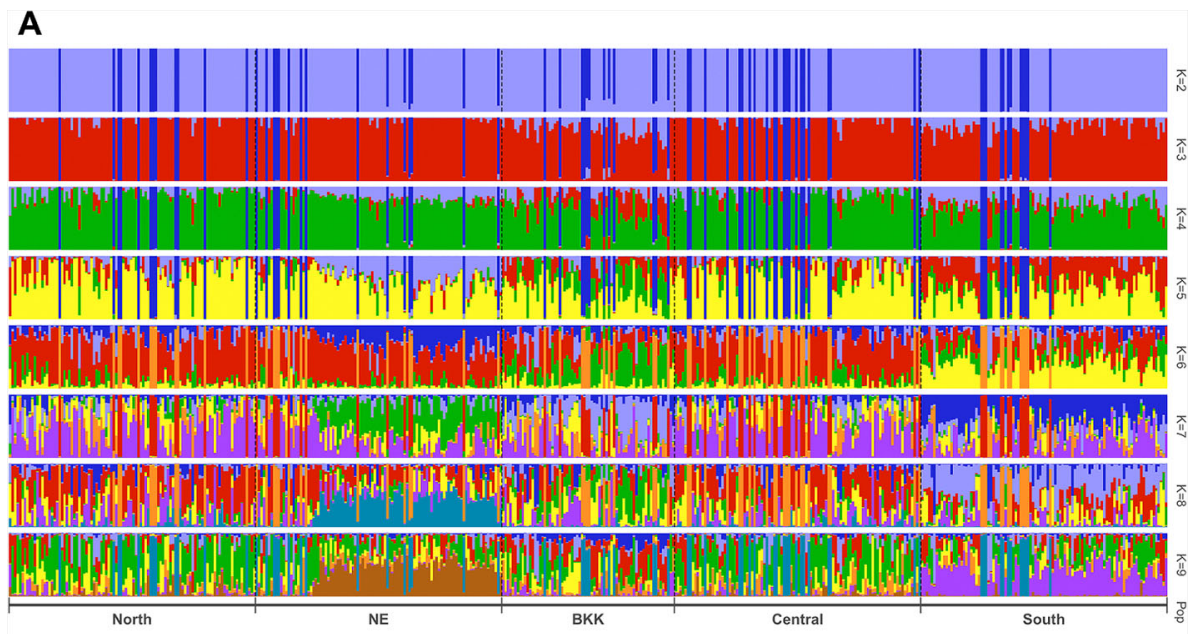

B

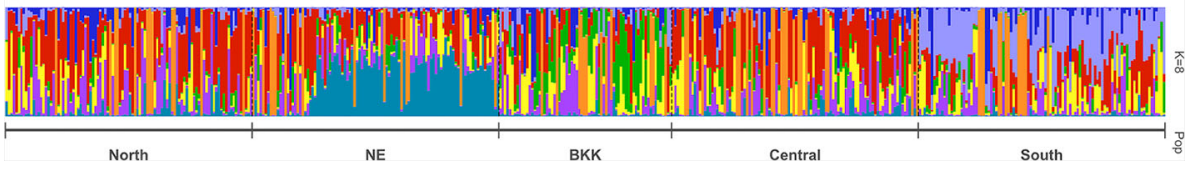

FIGURE 3 | Estimated population structure with z-score as the input based on STRUCTURE analysis with pophelper visualization tool. Bar plot of individual ancestry proportions for the genetic clusters inferred using (A) $K=2$ to 9 , and $(\mathbf{B})$ optimal $K=8$.

Abacavir is used for treatment of human immunodeficiency virus type I infection (Sukasem et al., 2014; White et al., 2014). Abacavir-hypersensitivity reaction (ABC-HSR) is a major of adverse drug reactions, and its reaction onset occurs during the first 6 weeks of treatment (Chaponda and Pirmohamed, 2011). Previous studies showed the association of $H L A-B^{\star} 57: 01$ allele with ABC-HSR in European and African (Mallal et al., 2008; Sukasem et al., 2014; White et al., 2014). The data from 470 Thais showed that the allele frequency of $H L A-B^{\star 57: 01}$ was not so common $(1.17 \%$ of 470): $1.4 \%$ Bangkok, $1.5 \%$ Central, $1 \%$ Northeastern, $1 \%$ Northern, and $1 \%$ Southern. Our results confirmed the previous study of 986 Thais which revealed $1.5 \%$ of $H L A-B^{\star} 57: 01$ allele distribution in Thailand (Puangpetch et al., 2015). The range of $H L A-B^{\star} 57: 01$ allele distribution was more common $(0.97 \%-4.15 \%)$ in African Americans, Caucasians, Hispanics, North American, and Asians (Cao et al., 2001). The distribution of Thai $H L A-B{ }^{\star 57: 01}$ allele was closer to Southeast Asian populations: $2.9 \%$ of 170 Vietnamese and $1.27 \%$ of 237 Indonesian (Hoa et al., 2008; Yuliwulandari et al., 2009). Thus, the allele frequency of $H L A-B^{\star 57: 01}$ has confirmed the value of the screening among different ethnicities before initiation of treatment.

CBZ-induced SJS/TEN was strongly associated with $H L A-B^{\star}$ 15:02 in Han Chinese, Thais, Vietnamese, Malaysian, and Indian (Ferrell and McLeod, 2008; Locharernkul et al., 2008; Hung et al., 2010; Chang et al., 2011; Nguyen et al., 2015). We found the allele frequency of $H L A-B^{\star} 15$ :02 was 0.0766 in Thais, especially, among individuals from Southern Thailand. Particularly, the high expression of HLA-B75 family distribution (HLA- $B^{\star} 15: 02,-B^{\star} 15: 11$, and $\left.H L A-B^{\star} 15: 21\right)$ in the Southern group was a genetic factor associated with CBZ-induced SJS/TEN. Nevertheless, the distribution of $H L A-B^{\star} 15: 02$ allele in
Caucasian and Japanese populations is less than $1 \%$ and African Americans is $0.20 \%$ (Amstutz et al., 2014; White et al., 2014). Therefore, $H L A-B^{\star}$ 15:02 allele is quite ethnic specific as well as having phenotype of SCARs. Furthermore, other studies supported $H L A-A^{\star} 31: 01$ associated with CBZ-induced SJS/TEN, DRESS, and maculopapular exanthema (MPE) in European, Japanese, Taiwan Han Chinese, and Korean (McCormack et al., 2011; Ozeki et al., 2011; Amstutz et al., 2014). The allele frequency of $H L A-A * 31: 01$ was higher among North Americans (7.75\%), Hispanics (4.91\%), and Asians (3.06\%), while Thais' and African Americans' were much lower, $0.85 \%$ and $0.79 \%$, respectively (Cao et al., 2001). The report also shows that $H L A-A^{\star} 31: 01$ is more common in most ethnic groups (Amstutz et al., 2014). The discordant allele frequencies in which Thais harbor less common $H L A-A^{\star} 31$ :01 allele supports the need to study more ethnic-specific $H L A$ alleles among Southeast Asian populations. Additionally, the cross-reactivity between PHTinduced SJS/TEN and OXC-induced SJS/TEN was associated with HLA- $B^{\star}$ 15:02 among Han Chinese (Hu et al., 2011; Su et al., 2016). Previous studies showed that CBZ-, PHT-, and LTG-induced SJS/ TEN and MPE were associated with $H L A-A^{\star} 24$ :02 (Moon et al., 2015; Shi et al., 2017). We found the distribution of $H L A-A^{\star} 24: 02$ allele was 0.1149 in Thailand which was also with similar rate across all five specific regions in Thailand. Although $H L A-A^{\star} 24: 02$ allele frequency in African Americans was much lower (0.0278), this HLA allele was used as a predictive marker for antiepileptic drug-induced SJS/TEN and MPE (Cao et al., 2001). The frequency distributions of pharmacogenetics markers from the neighboring countries were quite similar to Thais', including Vietnamese: $H L A-B^{\star} 15: 02(0.135)$ and $H L A-A^{\star} 24: 02$ (0.138), Indonesian: $H L A-B^{\star} 15: 02$ (0.116) and $H L A-A^{\star 24: 02}$ (0.143), Myanmar: $H L A-B^{\star} 15: 02$ (0.088) and HLA- 
$A^{\star} 24: 02$ (0.168), and Malaysian: $H L A-B^{\star} 15: 02$ (0.0825) (Hoa et al., 2008; Yuliwulandari et al., 2009; Jinam et al., 2010; Kongmaroeng et al., 2015). Similarly, the association between frequency of specific $H L A$ alleles and antiepileptic drug-induced SCARs was investigated in many populations.

Allopurinol-induced SJS/TEN causes disease spectrum, including skin rashes, fever, vasculitis, hepatitis, and epidermal necrosis (Lupton and Odom, 1979; Halevy et al., 2008; Tassaneeyakul et al., 2009). For Thai patients, HLA-B ${ }^{\star 58: 01}$ is strongly associated with allopurinol-induced SJS/TEN (odds ratio $=579.0 ; 95 \%$ CI: 29.5-11362.7; p-value < 0.001), DRESS (odds ratio $=430.3 ; 95 \% \mathrm{CI}: 22.6-8958.9 ;$ p-value $<0.001$ ), and MPE (odds ratio = 144.0; 95\% CI: 13.9-1497.0; p-value $<0.001$ ) (Sukasem et al., 2016). HLA-B ${ }^{\star 58: 01}$ was reported to be present $8 \%-15 \%$ among Han Chinese and Thai population, $0.6 \%$ of Japanese, and $0.8 \%$ of European population (Hung et al., 2005; Kaniwa et al., 2008; Tassaneeyakul et al., 2009; Somkrua et al., 2011). In this study, the frequency of $H L A-B^{\star} 58: 01$ allele was 0.0638 in 470 Thai population, ranging between 0.0500 and 0.0800 when considering at the regional group level. Note that the frequency of $H L A-B^{\star} 58: 01$ allele in Thailand was not much different when compared with other populations such as African Americans, Caucasians, Hispanics, North American, Asians, and Southeast Asians (Malaysia, Vietnam, Indonesia, and Myanmar) (Cao et al., 2001; Hoa et al., 2008; Yuliwulandari et al., 2009; Jinam et al., 2010; Kongmaroeng et al., 2015). Therefore, it is likely that the distribution of $H L A-B^{\star 58: 01}$ could be used as a universal pharmacogenetic marker for allopurinol-induced CADRs including SJS/TEN, DRESS, and MPE for all ethnicities.

Reports showed the association among Han Chinese leprosy patients and Thai non-leprosy patients between $H L A-B^{\star} 13: 01$ and dapsone hypersensitivity reactions (odds ratio 122.1, pvalue $=6.03810^{-12}$ and odds ratio 20.53, p-value $=6.84$ $10^{-25}$ ) and dapsone-induced SCARs (odds ratio 54.00, pvalue $=0.0001$ ) and dapsone-induced DRESS (odds ratio 60.75 , p-value $=0.0001$ ), respectively (Wang et al., 2013; Zhang et al., 2013; Tempark et al., 2017). The distribution of $H L A-B^{\star} 13: 01$ allele was absent in Europeans and Africans (Cao et al., 2001; Zhang et al., 2013). Recent publications showed varying distributions of $H L A-B^{\star} 13: 01$, including $1.5 \%$ of Japanese, $1 \%-12 \%$ of Indian, $28 \%$ of Papuans and Australian aborigines, $2 \%-20 \%$ of Chinese, $2 \%-4 \%$ of Southeast Asians, $1.94 \%$ of Koreans, and $6.95 \%$ of the previously reported Thai population (Cao et al., 2001; Zhang et al., 2013; Puangpetch et al., 2015; Tempark et al., 2017). This study showed that the frequency of $H L A-B^{\star} 13: 01$ allele was 0.0596 which is similar to the previous study in Thailand (Puangpetch et al., 2015). Furthermore, the frequency of $H L A-B^{\star} 13: 01$ allele was shown to have a very strong association with many drug-induced DRESS (PHT, phenobarbital, dapsone, and salazosulfapyridine) and Asian populations (Thais, Han Chinese, Malaysian, Vietnamese, Indonesian, and Myanmarese) (Hoa et al., 2008; Yuliwulandari et al., 2009; Jinam et al., 2010; Kongmaroeng et al., 2015). Interestingly, dapsone shows similarity in chemical structure to the sulfonamides; the cross-reactivity of sulfonamide hypersensitivity reactions was also reported
(Tomecki and Catalano, 1981). Co-trimoxazole (sulfonamide and trimethoprim) is a sulfonamide antibiotic and the most common culprit drug for SJS/TEN in many countries including Thailand (Barvaliya et al., 2011; Kongpan et al., 2015). The study reported the association of the alleles $H L A-B^{\star} 38: 01$ and $H L A$ $B^{\star}$ 38:02 with sulfamethoxazole-induced SJS/TEN in European patients (Lonjou et al., 2008). Furthermore, HLA- $B^{\star}$ 15:02 allele was found to be associated with co-trimoxazole-induced SJS/ TEN in Thais (odds ratio 3.91, p-value $=0.0037$ ) (Kongpan et al., 2015). In this study, we found the frequencies of $H L A-B^{\star} 38: 01$ allele was $0.000, H L A-B^{\star} 38$ :02 allele was 0.043 , and $H L A-B^{\star}$ 15:02 allele was 0.077 . With the presence of these alleles, further investigation should be conducted on other HLA alleles and co-trimoxazole-induced SCARs in Thai population.

Drug-induced liver injury (DILI) is rare, but it potentially causes serious idiosyncratic reaction (Kaplowitz, 2005). Previous study reported the association between amoxicillin-clavulanateinduced liver injury and $H L A$ haplotypes: $H L A-D R B 1^{\star} 15: 01$, $H L A-D Q B 1^{\star} 06: 02$, and $H L A-A^{\star} 30: 02$ (Stephens et al., 2013). In this study, we found that the frequencies of $H L A-D R B 1^{\star} 15: 01$ and $H L A-D Q B 1^{*} 06: 02$ were 0.0809 and 0.0149, respectively. However, $H L A-A^{\star}$ 30:02 allele was absent in Thai population. We noticed that the distribution of $H L A-D Q B 1^{\star} 06: 02$ allele in Thai population was much lower than those found in African Americans and Caucasians (Begovich et al., 1992; Just et al., 1996). The distribution of $H L A-D Q B 1^{\star} 06: 02$ was quite similar among Southeast Asian countries (Malaysia, Vietnam, and Myanmar) and was similar to Thais (Hoa et al., 2008; Jinam et al., 2010; Kongmaroeng et al., 2015). Additionally, the frequency of a pharmacogenetic marker associated with

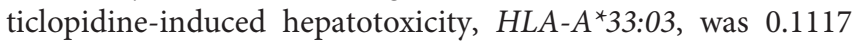
reported by our $H L A$ study of Thai cohort. Similarly, the frequencies of this pharmacogenetics marker were 0.1150 for Vietnamese, 0.1516 for Indonesian, 0.1380 for Burmese, and 0.08 for Malaysian (Hoa et al., 2008; Yuliwulandari et al., 2009; Jinam et al., 2010; Kongmaroeng et al., 2015). According to trends of $H L A$ prevalence of Southeast Asian, both $H L A-A^{\star} 33: 03$ and $H L A-D Q B 1^{*} 06: 02$ allele should be further investigated in which they can be used as pharmacogenetic markers in Thais and other neighboring populations.

Previous report demonstrated that Thai population contains four prominent substructures using 435,503 single-nucleotide polymorphisms (SNPs) collected from two independent studies comprising 992 Thai individuals (Wangkumhang et al., 2013). The analysis from their work revealed two important concepts: 1) the three main subpopulations are localized in Northern, Northeastern, and Southern parts of Thailand while the members from the fourth group are scattered throughout the country and 2) place of origins of Thai individuals might be discordant with the genetic similarity profile of that place. In other words, people from the north could migrate to the south and stay there for more than three generations. Similar findings were shown in our population genetic analyses in which Northeastern and Southern groups were genetically different while Bangkok and Central groups were mixed and/or scattered to Northern, Northeastern, and Southern groups. In 
terms of $H L A$-haplotype distributions over the five regions, we found that the haplotype frequencies in these five regions were slightly different. However, we did not observe novel HLAhaplotypes specific to any subregion. The PCR probes used to call these HLA-CLASS I and CLASS II rely on some known collections of HLAs and might not be able to predict novel HLA haplotypes.

Ethnic-specific genetic variation database is vital to identification of good pharmacogenetic markers in Asian countries such as CYP2C9*3 associated with PHT-induced SJS/ TEN in Taiwanese, Japanese, and Malaysians (Chung et al., 2014). Further studies should be done to confirm pharmacogenetics markers from the ethnic-specific SNP databases. Since there were only six haplotypes and 470 individuals, the challenge of this study was the data analyses obtained from the platform called PCR-SSO probe. To address the lack of genetic polymorphisms in our population genetic study, we extracted probe signals laid across six stretches of HLA. Using the probe signals, we observed some distinct as well as cline subpopulations. This discrepancy should be clarified in further study by performing high-resolution DNA typing and recruiting more Thai individuals. In this study, we identified both HLA class I and II alleles in Thai population. Furthermore, many $H L A$ class I and II alleles were associated with pharmacogenetics markers which might appear exclusively in many populations. Particularly, a database containing distribution of specific HLA class I and II alleles will support the development of the pharmacogenetics markers for screening drug hypersensitivity reactions.

\section{DATA AVAILABILITY STATEMENT}

The datasets used in this study can be found here https://www. ebi.ac.uk/ipd/imgt/hla using the accession numbers HLAA*31:01 (IMGT/HLA ID: HLA00097), HLA-A*33:03 (IMGT/HLA ID: HLA00106), HLA-B ${ }^{\star} 13: 01$ (IMGT/HLA ID: HLA00152), HLA-B ${ }^{\star}$ 15:02 (IMGT/HLA ID: HLA00165), HLA$\mathrm{B} * 15: 08$ (IMGT/HLA ID: HLA00171), HLA-B*15:11 (IMGT/HLA ID: HLA00174), HLA-B*15:21 (IMGT/HLA ID: HLA00184), HLA-B³5:05 (IMGT/HLA ID: HLA00241), HLAB*57:01(IMGT/HLA ID: HLA00381), HLA-B*58:01 (IMGT/HLA ID: HLA00386), HLA-B ${ }^{\star 59: 01 ~(I M G T / H L A ~ I D: ~}$ HLA00389), HLA-C ${ }^{\star}$ 03:02 (IMGT/HLA ID: HLA00410), HLA-

\section{REFERENCES}

Aihara, M. (2011). Pharmacogenetics of cutaneous adverse drug reactions. J. Dermatol. 38, 246- 254. doi: 10.1111/j.1346-8138.2010.01196.x

Amstutz, U., Shear, N. H., Rieder, M. J., Hwang, S., Fung, V., Nakamura, H., et al. (2014). Recommendations for HLA-B* 15:02 and HLA-A*31:01 genetic testing to reduce the risk of carbamazepine-induced hypersensitivity reactions. Epilepsia 55, 496-506. doi: 10.1111/epi.12564

Anania, C., Olivero, F., Spagnolo, A., Chiesa, C., and Pacifico, L. (2017). Immune response to vaccines in children with celiac disease. World J. Gastroenterol. 23, 3205-3213. doi: 10.3748/wjg.v23.i18.3205
$\mathrm{C}^{\star}$ 06:02 (IMGT/HLA ID: HLA00430), HLA-C*08:01 (IMGT/HLA ID: HLA00445), HLA-DRB1¹3:02 (IMGT/HLA ID: HLA00798), HLA-DRB1*15:02 (IMGT/HLA ID: HLA 00867) and HLA-DQB ${ }^{\star} 06: 02$ (IMGT/HLA ID: HLA00646).

\section{ETHICS STATEMENT}

The studies involving human participants were reviewed and approved by the Ethical Clearance Committee on Human Rights Related to Research Involving Human Subjects Faculty of Medicine Ramathibodi Hospital Mahidol University. The patients/participants provided their written informed consent to participate in this study.

\section{AUTHOR CONTRIBUTIONS}

PS had substantial contributions to the conception, design, analysis, and interpretation of the data, drafting the manuscript, and agrees to be accountable for all aspects of the work. PJ, TJ, NK, CC, JP, CNa, WA, ST, CN and AW had substantial contributions to the conception and analysis of the data and drafting the manuscript. CS had substantial contributions to the conception and design of the work, drafting the work, and agrees to be accountable for all aspects of the work in ensuring that questions related to the accuracy or integrity of any part of the work are appropriately investigated and resolved and provide approval for publication of the content.

\section{ACKNOWLEDGMENTS}

This study was supported by grants from the (1) Faculty of Medicine, Ramathibodi Hospital, Mahidol University (2) The National Health Examination Survey Office, Health System Research Institute. The authors thank the study participants and staff of Pharmacogenomic and Personalized Medicine of Ramathibodi Hospital, the Royal Golden Jubilee Ph.D. (RGJPHD) Program: PHD/0153/2559, and The International Research Network-The Thailand Research Fund (IRN60W003), Thailand.

Arnaiz-Villena,A.,Palacio-Grüber,J.,CamposCristina,E.M., Rubio,J.A.,Casado,E. G., etal. (2016). HLA genes in Chimila Amerindians (Colombia), the peopling of America and medical implications. Int. J. Mod. Anthrop. 9, 91-121. doi: 10.4314/ijma.v1i9.6

Barvaliya, M., Sanmukhani, J., Patel, T., Paliwal, N., Shah, H., and Tripathi, C. (2011). Drug-induced Stevens-Johnson syndrome (SJS), toxic epidermal necrolysis (TEN), and SJS-TEN overlap: a multicentric retrospective study. J. Postgrad. Med. 57, 115-119. doi: 10.4103/0022-3859.81865

Begovich, A. B., McClure, G. R., Suraj, V. C., Helmuth, R. C., Fildes, N., Bugawan, T. L., et al. (1992). Polymorphism, recombination, and linkage disequilibrium within the HLA class II region. J. Immunol. 148, 249-258. 
Cao, K., Hollenbach, J., Shi, X., Shi, W., Chopek, M., and Fernández-Viña, M. A. (2001). Analysis of the frequencies of HLA-A, B, and C alleles and haplotypes in the five major ethnic groups of the United States reveals high levels of diversity in these loci and contrasting distribution patterns in these populations. Hum. Immunol. 62, 1009-1030. doi: 10.1016/S0198-8859(01) 00298-1

Chaichompoo, K., Abegaz, F., Tongsima, S., Shaw, P. J., Sakuntabhai, A., Pereira, L., et al. (2018). KRIS: Keen and Reliable Interface Subroutines for bioinformatics analysis. R. Package version 1.1.1. Available at: https:/CRAN.R-project.org/ package=KRIS. (Accessed 5 December 2018).

Chang, C. C., Too, C. L., Murad, S., and Hussein, S. H. (2011). Association of HLA-B ${ }^{\star} 1502$ allele with carbamazepine-induced toxic epidermal necrolysis and Stevens-Johnson syndrome in the multi-ethnic Malaysian population. Int. J. Dermatol. 50, 221-224. doi: 10.1111/j.1365-4632.2010.04745.X

Chang, C. C., Ng, C. C., Too, C. L., Choon, S. E., Lee, C. K., Chung, W. H., et al. (2017). Association of HLA-B*15:13 and HLA-B*15:02 with phenytoininduced severe cutaneous adverse reactions in a Malay population. Pharmacogenomics J. 17, 170-173. doi: 10.1038/tpj.2016.10

Chantarangsu, S., Mushiroda, T., Mahasirimongkol, S., Kiertiburanakul, S., Sungkanuparph, S., Manosuthi, W., et al. (2009). HLA-B ${ }^{\star} 3505$ allele is a strong predictor for nevirapine-induced skin adverse drug reactions in HIVinfected Thai patients. Pharmacogenet. Genomics 19, 139-146. doi: 10.1097/ FPC.0b013e32831d0faf

Chaponda, M., and Pirmohamed, M. (2011). Hypersensitivity reactions to HIV therapy. Br. J. Clin. Pharmacol. 71, 659-671. doi: 10.1111/j.13652125.2010.03784.x

Chung, W. H., Chang, W. C., Lee, Y. S., Wu, Y. Y., Yang, C. H., Ho, H. C., et al. (2014). Genetic variants associated with phenytoin-related severe cutaneous adverse reactions. JAMA 312, 525-534. doi: 10.1001/jama.2014.7859

Criado, P. R., Criado, R. F., Avancini, J. M., and Santi, C. G. (2012). Drug Reaction with Eosinophilia and Systemic Symptoms (DRESS)/Drug-Induced Hypersensitivity Syndrome (DIHS): a review of current concepts. Bras. Dermatol. 87, 435-449. doi: 10.1590/S0365-05962012000300013

Cristallo, A. F., Schroeder, J., Citterio, A., Santori, G., Ferrioli, G. M., Rossi, U., et al. (2011). A study of HLA class I and class II 4-digit allele level in StevensJohnson syndrome and toxic epidermal necrolysis. Int. J. Immunogenet. 38, 303-309. doi: 10.1111/j.1744-313X.2011.01011.x

Daly, A. K., Donaldson, P. T., Bhatnagar, P., Shen, Y., Pe'er, I., Floratos, A., et al. (2009). HLA-B ${ }^{\star} 5701$ genotype is a major determinant of drug-induced liver injury due to flucloxacillin. Nat. Genet. 41, 816-819. doi: 10.1038/ng.379

Dao, R. L., Su, S. C., and Chung, W. H. (2015). Recent advances of pharmacogenomics in severe cutaneous adverse reactions: immune and nonimmune mechanisms. Asia Pac. Allergy 5, 59-67. doi: 10.5415/ apallergy.2015.5.2.59

Donnell PH, O., and Dolan, M. E. (2009). Cancer pharmacoethnicity: ethnic differences in susceptibility to the effects of chemotherapy. Clin. Cancer Res. 15, 4806-4814. doi: 10.1158/1078-0432.CCR-09-0344

Evanno, G., Regnaut, S., and Goudet, J. (2005). Detecting the number of clusters of individuals using the software STRUCTURE: a simulation study. Mol. Ecol. 14, 266-2620. doi: 10.1111/j.1365-294X.2005.02553.x

Ferrell, P. B.Jr, and McLeod, H. L. (2008). Carbamazepine, HLA-B ${ }^{\star} 1502$ and risk of Stevens-Johnson syndrome and toxic epidermal necrolysis: US FDA recommendations. Pharmacogenomics 9, 1543-1546. doi: 10.2217/ 14622416.9.10.1543

Francis, R. M. (2017). pophelper: an R package and web app to analyse and visualise population structure. Mol. Ecol. Resour. 17 (1), 27-32. doi: 10.1111/ 1755-0998.12509. https://github.com/royfrancis/pophelper. pophelper R package version 2.3.0

Halevy, S., Ghislain, P. D., Mockenhaupt, M., Fagot, J. P., Bouwes Bavinck, J. N., Sidoroff, A., et al. (2008). Allopurinol is the most common cause of StevensJohnson syndrome and toxic epidermal necrolysis in Europe and Israel. J. Am. Acad. Dermatol. 58, 25-32. doi: 10.1016/j.jaad.2007.08.036

Hautekeete, M. L., Horsmans, Y., Van Waeyenberge, C., Demanet, C., Henrion, J., Verbist, L., et al. (1999). HLA association of amoxicillin-clavulanate-induced hepatitis. Gastroenterology 117, 1181-1186. doi: 10.1016/S0016-5085(99) 70404-X

Hiransuthikul, A., Rattananupong, T., Klaewsongkram, J., Rerknimitr, P., Pongprutthipan, M., and Ruxrungtham, K. (2016). Drug-induced hypersensitivity syndrome/drug reaction with eosinophilia and systemic symptoms (DIHS/DRESS): 11 years retrospective study in Thailand. Allergol. Int. 65, 432-438. doi: 10.1016/j.alit.2016.04.001

Hirata, K., Takagi, H., Yamamoto, M., Matsumoto, T., Nishiya, T., Mori, K., et al. (2008). Ticlopidine-induced hepatotoxicity is associated with specific human leukocyte antigen genomic subtypes in Japanese patients: a preliminary casecontrol study. Pharmacogenomics J. 8, 29-33. doi: 10.1038/sj.tpj.6500442

Hoa, B. K., Hang, N. T., Kashiwase, K., Ohashi, J., Lien, L. T., Horie, T., et al. (2008). HLA-A, -B, -C, -DRB1 and -DQB1 alleles and haplotypes in the Kinh population in Vietnam. Tissue Antigens 71, 127-134. doi: 10.1111/j.13990039.2007.00982.x

Howell, W. M., Carter, V., and Clark, B. (2010). The HLA system: immunobiology, HLA typing, antibody screening and crossmatching techniques. J. Clin. Pathol. 63, 387-390. doi: 10.1136/jcp.2009.072371

Hu, F. Y., Wu, X. T., An, D. M., Yan, B., Stefan, H., and Zhou, D. (2011). Pilot association study of oxcarbazepine-induced mild cutaneous adverse reactions with HLA-B ${ }^{\star} 1502$ allele in Chinese Han population. Seizure 20, 160-162. doi: 10.1016/j.seizure.2010.11.014

Hung, S. I., Chung, W. H., Liou, L. B., Chu, C. C., Lin, M., Huang, H. P., et al. (2005). HLA-B ${ }^{\star} 5801$ allele as a genetic marker for severe cutaneous adverse reactions caused by allopurinol. Proc. Natl. Acad. Sci. U. S. A. 102, 4134-4139. doi: $10.1073 /$ pnas.0409500102

Hung, S. I., Chung, W. H., Liu, Z. S., Chen, C. H., Hsih, M. S., Hui, R. C., et al. (2010). Common risk allele in aromatic antiepileptic-drug induced StevensJohnson syndrome and toxic epidermal necrolysis in Han Chinese. Pharmacogenomics 11, 349-356. doi: 10.2217/pgs.09.162

Iasella, C. J., Johnson, H. J., and Dunn, M. A. (2017). Adverse drug reactions: type A (Intrinsic) or type B (Idiosyncratic). Clin. Liver Dis. 21, 73-87. doi: 10.1016/ j.cld.2016.08.005

Illing, P. T., Purcell, A. W., and McCluskey, J. (2017). The role of HLA genes in pharmacogenomics: unravelling HLA associated adverse drug reactions. Immunogenetics 10, 1-14. doi: 10.1007/s00251-017-1007-5

In, J. W., Roh, E. Y., Oh, S., Shin, S., Park, K. U., and Song, E. Y. (2015). Allele and Haplotype frequencies of human Leukocyte Antigen-A, -B, -C, -DRB1, and -DQB1 from sequence-based DNA typing data in Koreans. Ann. Lab. Med. 35, 429-435. doi: 10.3343/alm.2015.35.4.429

Jaruthamsophon, K., Sripo, T., Sukasem, C., and Limprasert, P. (2016). Comparison of a new in-house and three published HLA-B ${ }^{\star}$ 15:02 screening methods for prevention of Carbamazepine-induced severe drug reactions. PloS One 11, e0155907. doi: 10.1371/journal.pone.0155907

Jinam, T. A., Saitou, N., Edo, J., Mahmood, A., and Phipps, M. E. (2010). Molecular analysis of HLA Class I and Class II genes in four indigenous Malaysian populations. Tissue Antigens 75, 151-158. doi: 10.1111/j.1399. 0039.2009.01417.x

Just, J. J., King, M. C., Thomson, G., and Klitz, W. (1996). African-American HLA class II allele and haplotype diversity. Tissue Antigens 48, 636-644. doi: 10.1111/j.1399-0039.1996.tb02686.x

Kaniwa, N., Saito, Y., Aihara, M., Matsunaga, K., Tohkin, M., and Kurose, K. (2008). HLA-B locus in Japanese patients with anti-epileptics and allopurinolrelated Stevens-Johnson syndrome and toxic epidermal necrolysis. Pharmacogenomics 9, 1617-1622. doi: 10.2217/14622416.9.11.1617

Kaplowitz, N. (2005). Idiosyncratic drug hepatotoxicity. Nat. Rev. Drug Discovery 4, 489-499. doi: 10.1038/nrd1750

Kazeem, G. R., Cox, C., Aponte, J., Messenheimer, J., Brazell, C., Nelsen, A. C., et al. (2009). High-resolution HLA genotyping and severe cutaneous adverse reactions in lamotrigine-treated patients. Pharmacogenet. Genomics 19, 661665. doi: 10.1097/FPC.0b013e32832c347d

Kim, B. K., Jung, J. W., Kim, T. B., Chang, Y. S., Park, H. S., Moon, J., et al. (2017). HLA-A ${ }^{\star} 31: 01$ and lamotrigine-induced severe cutaneous adverse drug reactions in a Korean population. Ann. Allergy Asthma Immunol. 118, 629-630. doi: 10.1016/ j.anai.2017.02.011

Kongmaroeng, C., Romphruk, A., Puapairoj, C., Leelayuwat, C., Kulski, J. K., Inoko, H., et al. (2015). HLA alleles and haplotypes in Burmese (Myanmarese) and Karen in Thailand. Tissue Antigens 86, 199-204. doi: 10.1111/tan.12637

Kongpan, T., Mahasirimongkol, S., Konyoung, P., Kanjanawart, S., Chumworathayi, P., Wichukchinda, N., et al. (2015). Candidate HLA genes for prediction of co-trimoxazole-induced severe cutaneous reactions. Pharmacogenet. Genomics 25, 402-411. doi: 10.1097/FPC.0000000000000153 
Kopelman, N. M., Mayzel, J., Jakobsson, M., Rosenberg, N. A., and Mayrose, I. (2015). Clumpak: a program for identifying clustering modes and packaging population structure inferences across K. Mol. Ecol. Resour. 15 (5), 1179-1191. doi: 10.1111/1755-0998.12387

Lauren, W., Vincent, Y., and Munir, P. (2014). "Adverse drug reactions," in Handbook of pharmacogenomics and stratified medicine. Ed. S. Padmanabhan (London: Academic press), 405-435.

Lee, H. Y., Martanto, W., and Thirumoorthy, T. (2013). Epidemiology of StevensJohnson syndrome and toxic epidermal necrolysis in Southeast Asia. Dermatol. Sin. 31, 217-220. doi: 10.1016/j.dsi.2013.09.007

Li, X., Zhao, Z., and Sun, S. S. (2017). Association of human leukocyte antigen variants and allopurinol-induced Stevens-Johnson syndrome and toxic epidermal necrolysis: a meta-analysis. Am. J. Health Syst. Pharm. 74, e183e192. doi: 10.2146/ajhp160243

Locharernkul, C., Loplumlert, J., Limotai, C., Korkij, W., Desudchit, T., Tongkobpetch, S., et al. (2008). Carbamazepine and phenytoin induced Stevens-Johnson syndrome is associated with HLA-B ${ }^{*} 1502$ allele in Thai population. Epilepsia 49, 2087-2091. doi: 10.1111/j.1528-1167.2008.01719.x

Lonjou, C., Borot, N., Sekula, P., Ledger, N., Thomas, L., Halevy, S., et al. (2008). A European study of HLA-B in Stevens-Johnson syndrome and toxic epidermal necrolysis related to five high-risk drugs. Pharmacogenet. Genomics 18, 99-107. doi: 10.1097/FPC.0b013e3282f3ef9c

Lucena, M. I., Molokhia, M., Shen, Y., Urban, T. J., Aithal, G. P., Andrade, R. J., et al. (2011). Susceptibility to amoxicillin-clavulanate-induced liver injury is influenced by multiple HLA class I and II alleles. Gastroenterology 141, 338-347. doi: 10.1053/ j.gastro.2011.04.001

Lupton, G. P., and Odom, R. B. (1979). The allopurinol hypersensitivity syndrome. J. Am. Acad. Dermatol. 1, 365-374. doi: 10.1016/S0190-9622(79)70031-4

Lv, Y. D., Min, F. L., Liao, W. P., He, N., Zeng, T., Ma, D. H., et al. (2013). The association between oxcarbazepine-induced maculopapular eruption and HLA-B alleles in a Northern Han Chinese population. BMC Neurol. 13, 1-6. doi: 10.1186/1471-2377-13-75

Mallal, S., Phillips, E., Carosi, G., Molina, J. M., Workman, C., Tomazic, J., et al. (2008). HLA-B ${ }^{\star} 5701$ screening for hypersensitivity to abacavir. N. Engl. J. Med. 358, 568-579. doi: 10.1056/NEJMoa0706135

Manuyakorn, W., Mahasirimongkol, S., Likkasittipan, P., Kamchaisatian, W., Wattanapokayakit, S., Inunchot, W., et al. (2016). Association of HLA genotypes with phenobarbital hypersensitivity in children. Epilepsia 57, 1610-1616. doi: 10.1111/epi.13509

Marotti, M. (2012). Severe cutaneous adverse reactions (SCAR) syndromes. Rev. Assoc. Med. Bras. 58, 276-278. doi: 10.1590/S0104-42302012000300003

McCormack, M., Alfirevic, A., Bourgeois, S., John, J. F., Kasperaviciute, D., Carrington, M., et al. (2011). HLA-A ${ }^{\star} 3101$ and carbamazepine-induced hypersensitivity reactions in Europeans. N. Engl. J. Med. 364, 1134-1143. doi: 10.1056/NEJMoa1013297

Moon, J., Park, H. K., Chu, K., Sunwoo, J. S., Byun, J. I., Lim, J. A., et al. (2015). The HLA-A ${ }^{\star} 2402 / \mathrm{Cw}^{\star} 0102$ haplotype is associated with lamotrigine-induced maculopapular eruption in the Korean population. Epilepsia 56, 161-167. doi: 10.1111/epi.13087

Nahoko, K., and Yoshiro, S. (2013). The risk of cutaneous adverse reactions among patients with the HLA-A* 31:01 allele who are given carbamazepine, oxcarbazepine or eslicarbazepine: a perspective review. Ther. Adv. Drug Saf. 4, 246-253. doi: 10.1177/2042098613499791

Nguyen, D. V., Chu, H. C., Nguyen, D. V., Phan, M. H., Craig, T., Baumgart, K., et al. (2015). HLA-B ${ }^{\star} 1502$ and carbamazepine-induced severe cutaneous adverse drug reactions in Vietnamese. Asia Pac. Allergy 5, 68-77. doi: 10.5415/apallergy.2015.5.2.68

Ozeki, T., Mushiroda, T., Yowang, A., Takahashi, A., Kubo, M., Shirakata, Y., et al. (2011). Genome-wide association study identifies HLA-A ${ }^{\star 3101}$ allele as a genetic risk factor for carbamazepine-induced cutaneous adverse drug reactions in Japanese population. Hum. Mol. Genet. 20, 1034-1041. doi: 10.1093/hmg/ddq537

Petersdorf, E. W. (2017). Role of major histocompatibility complex variation in graft-versus-host disease after hematopoietic cell transplantation. F1000Res 3 , 1-12. doi: 10.12688/f1000research.10990.1

Pritchard, J. K., Stephens, M., and Donnelly, P. (2000). Inference of population structure using multilocus genotype data. Genetics 155 (2), 945-959.

Puangpetch, A., Koomdee, N., Chamnanphol, M., Jantararoungtong, T., Santon, S., Prommas, S., et al. (2015). HLA-B allele and haplotype diversity among Thai patients identified by PCR-SSOP: evidence for high risk of drug-induced hypersensitivity. Front. Genet. 478, 1-7. doi: 10.3389/fgene.2014.00478

Saito, S., Ota, S., Yamada, E., Inoko, H., and Ota, M. (2000). Allele frequencies and haplotypic associations defined by allelic DNA typing at HLA class I and class II loci in the Japanese population. Tissue Antigens 56, 522-529. doi: 10.1034/ j.1399-0039.2000.560606.x

Sette, A., and Sidney, J. (1998). HLA supertypes and supermotifs: a functional perspective on HLA polymorphism. Curr. Opin. Immunol. 10, 478-482. doi: 10.1016/S0952-7915(98)80124-6

Shi, Y. W., Min, F. L., Zhou, D., Qin, B., Wang, J., Hu, F. Y., et al. (2017). HLA$A^{\star} 24: 02$ as a common risk factor for antiepileptic drug-induced cutaneous adverse reactions. Neurology 88, 2183-2191. doi: 10.1212/WNL.000 0000000004008

Shiina, T., Hosomichi, K., Inoko, H., and Kulski, J. K. (2009). The HLA genomic loci map: expression, interaction, diversity and disease. J. Hum. Genet. 54, 15-39. doi: 10.1038/jhg.2008.5

Somkrua, R., Eickman, E. E., Saokaew, S., Lohitnavy, M., and Chaiyakunapruk, N. (2011). Association of HLA-B ${ }^{\star} 5801$ allele and allopurinol-induced Stevens Johnson syndrome and toxic epidermal necrolysis: a systematic review and meta-analysis. BMC Med. Genet. 12, 1-10. doi: 10.1186/1471-2350-12-118

Somkrua, R., Eickman, E. E., Saokaew, S., Lohitnavy, M., and Chaiyakunapruk, N. (2011). Association of HLA-B ${ }^{\star} 5801$ allele and allopurinol-induced Stevens Johnson syndrome and toxic epidermal necrolysis: a systematic review and meta-analysis. BMC Med. Genet. 12, 1-10. doi: 10.1186/1471-2350-12-118

Spraggs, C. F., Budde, L. R., Briley, L. P., Bing, N., Cox, C. J., King, K. S., et al. (2011). HLA-DQA $1^{*} 02: 01$ is a major risk factor for lapatinib-induced hepatotoxicity in women with advanced breast cancer. J. Clin. Oncol. 29, 667-673. doi: 10.1200/JCO.2010.31.3197

Stephens, C., López-Nevot, MÁ, Ruiz-Cabello, F., Ulzurrun, E., Soriano, G., Romero-Gómez, M., et al. (2013). HLA alleles influence the clinical signature of amoxicillin-clavulanate hepatotoxicity. PloS One 8, e68111. doi: 10.1371/ journal.pone.0068111

Su, S. C., Hung, S. I., Fan, W. L., Dao, R. L., and Chung, W. H. (2016). Severe cutaneous adverse reactions: the pharmacogenomics from research to clinical implementation. Int. J. Mol. Sci. 17, 1-10. doi: 10.3390/ijms17111890

Sukasem, C., Puangpetch, A., Medhasi, S., and Tassaneeyakul, W. (2014). Pharmacogenomics of drug-induced hypersensitivity reactions: challenges, opportunities and clinical implementation. Asian Pac. J. Allergy Immunol. 32, 111-123.

Sukasem, C., Jantararoungtong, T., Kuntawong, P., Puangpetch, A., Koomdee, N., Satapornpong, P., et al. (2016). HLA-B ${ }^{\star 58: 01}$ for allopurinol-induced cutaneous adverse drug reactions: implication for clinical interpretation in Thailand. Front. Pharmacol. 7, 1-8. doi: 10.3389/fphar.2016.00186

Tassaneeyakul, W., Jantararoungtong, T., Chen, P., Lin, P. Y., Tiamkao, S., Khunarkornsiri, U., et al. (2009). Strong association between HLA-B ${ }^{\star} 5801$ and allopurinol-induced Stevens-Johnson syndrome and toxic epidermal necrolysis in a Thai population. Pharmacogenet. Genomics 19, 704-709. doi: 10.1097/FPC.0b013e328330a3b8

Tempark, T., Satapornpong, P., Rerknimitr, P., Nakkam, N., Saksit, N., Wattanakrai, P., et al. (2017). Dapsone-induced severe cutaneous adverse drug reactions are strongly linked with HLA-B*13:01 allele in the Thai population. Pharmacogenet. Genomics 27, 429-437. doi: 10.1097/ FPC.0000000000000306

Tomecki, K. J., and Catalano, C. J. (1981). Dapsone hypersensitivity: the sulfone syndrome revisited. Arch. Dermatol. 117, 38-39. doi: 10.1001/ archderm.1981.01650010044023

Ulvestad, E., Williams, K., Bo, L., Trapp, B., Antel, J., and Mork, S. (1994). HLA class II molecules (HLA-DR, -DP, -DQ) on cells in the human CNS studied in situ and in vitro. Immunology 82, 535-541.

Wang, H., Yan, L., Zhang, G., Chen, X., Yang, J., Li, M., et al. (2013). Association between HLA- $\mathrm{B}^{\star} 1301$ and dapsone-induced hypersensitivity reactions among leprosy patients in China. J. Invest. Dermatol. 133, 2642-2644. doi: 10.1038/ jid.2013.192

Wangkumhang, P., Shaw, P. J., Chaichoompu, K., Ngamphiw, C., Assawamakin, A., Nuinoon, M., et al. (2013). Insight into the peopling of mainland Southeast Asia from Thai population genetic structure. PloS One 8, e79522. doi: 10.1371/ journal.pone.0079522

White, K. D., Gaudieri, S., and Phillips, E. J. (2014). "HLA and the pharmacogenomics of drug hypersensitivity," in Handbook of 
pharmacogenomics and stratified medicine. Ed. S. Padmanabhan (London: Academic press), 437-465.

Yampayon, K., Sukasem, C., Limwongse, C., Chinvarun, Y., Tempark, T., Rerkpattanapipat, T., et al. (2017). Influence of genetic and nongenetic factors on phenytoin-induced severe cutaneous adverse drug reactions. Eur. J. Clin. Pharmacol. 73, 855-865. doi: 10.1007/ s00228-017-2250-2

Yang, F., Gu, B., Zhang, L., Xuan, J., Luo, H., Zhou, P., et al. (2014). HLA$B^{\star} 13: 01$ is associated with salazosulfapyridine-induced drug rash with eosinophilia and systemic symptoms in Chinese Han population. Pharmacogenomics 15, 1461-1469. doi: 10.2217/pgs.14.69

Yang, F., Xuan, J., Chen, J., Zhong, H., Luo, H., Zhou, P., et al. (2016). HLA$B^{\star}$ 59:01: a marker for Stevens-Johnson syndrome/toxic epidermal necrolysis caused by methazolamide in Han Chinese. Pharmacogenomics J. 16, 83-87. doi: 10.1038/tpj.2015.25

Yuliwulandari, R., Kashiwase, K., Nakajima, H., Uddin, J., Susmiarsih, T. P., Sofro, A. S., et al. (2009). Polymorphisms of HLA genes in Western Javanese (Indonesia): close affinities to Southeast Asian populations. Tissue Antigens 73, 46-53. doi: 10.1111/j.1399-0039.2008.01178.x

Yun, J., Adam, J., Yerly, D., and Pichler, W. J. (2012). Human leukocyte antigens (HLA) associated drug hypersensitivity: consequences of drug binding to HLA. Allergy 67, 1338-1346. doi: 10.1111/all.12008
Zhang, F. R., Liu, H., Irwanto, A., Fu, X. A., Li, Y., Yu, G. Q., et al. (2013). HLA$\mathrm{B}^{\star} 13: 01$ and the dapsone hypersensitivity syndrome. N. Engl. J. Med. 369, 1620-1628. doi: 10.1056/NEJMoa1213096

Zhou, F., Cao, H., Zuo, X., Zhang, T., Zhang, X., Liu, X., et al. (2016). Deep sequencing of the MHC region in the Chinese population contributes to studies of complex disease. Nat. Genet. 48, 740-746. doi: 10.1038/ng.3576

Conflict of Interest: The authors declare that the research was conducted in the absence of any commercial or financial relationships that could be construed as a potential conflict of interest.

The reviewer TM declared a past co-authorship with one of the authors CS to the handling editor.

Copyright (c) 2020 Satapornpong, Jinda, Jantararoungtong, Koomdee, Chaichan, Pratoomwun, Na Nakorn, Aekplakorn, Wilantho, Ngamphiw, Tongsima and Sukasem. This is an open-access article distributed under the terms of the Creative Commons Attribution License (CC BY). The use, distribution or reproduction in other forums is permitted, provided the original author(s) and the copyright owner(s) are credited and that the original publication in this journal is cited, in accordance with accepted academic practice. No use, distribution or reproduction is permitted which does not comply with these terms. 